ARTICLE

https://doi.org/10.1038/s41467-019-14231-w

\title{
Competitive oxidation and ubiquitylation on the evolutionarily conserved cysteine confer tissue- specific stabilization of Insig-2
}

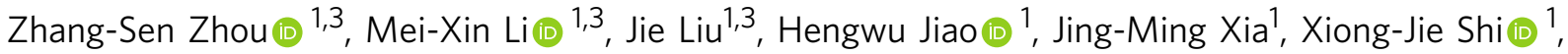 \\ Huabin Zhao (1) ${ }^{1}$, Liping $\mathrm{Chu}^{2}$, Jingrong $\mathrm{Liu}^{2}$, Wei Qi ${ }^{2}$, Jie Luo (i) ${ }^{1} \&$ Bao-Liang Song (i) ${ }^{1 \star}$
}

Insig-2 is an ER membrane protein negatively controlling lipid biosynthesis. Here, we find that Insig-2 is increased in the tissues, including liver, but unaltered in the muscle of $g p 78$ deficient mice. In hepatocytes and undifferentiated C2C12 myoblasts, Insig-2 is ubiquitylated on Cys 215 by gp78 and degraded. However, the $\mathrm{C} 215$ residue is oxidized by elevated reactive oxygen species (ROS) during $\mathrm{C} 2 \mathrm{C} 12$ myoblasts differentiating into myotubes, preventing Insig-2 from ubiquitylation and degradation. The stabilized Insig-2 downregulates lipogenesis through inhibiting the SREBP pathway, helping to channel the carbon flux to ATP generation and protecting myotubes from lipid over-accumulation. Evolutionary analysis shows that the YECK (in which C represents Cys215 in human Insig-2) tetrapeptide sequence in Insig-2 is highly conserved in amniotes but not in aquatic amphibians and fishes, suggesting it may have been shaped by differential selection. Together, this study suggests that competitive oxidation-ubiquitylation on Cys215 of Insig-2 senses ROS and prevents muscle cells from lipid accumulation.

\footnotetext{
${ }^{1}$ Hubei Key Laboratory of Cell Homeostasis, College of Life Sciences, Wuhan University, Wuhan 430072, China. ${ }^{2}$ School of Life Science and Technology, ShanghaiTech Universiy, Shanghai 201203, China. ${ }^{3}$ These authors contributed equally: Zhang-Sen Zhou, Mei-Xin Li, Jie Liu. *email: blsong@whu.edu.cn
} 
C holesterol, the most abundant sterol, is essential for membrane function in mammalian cells and its metabolism is tightly regulated. Cellular cholesterol can be synthesized from acetyl-CoA through the mevalonate pathway and obtained through receptor-mediated endocytosis of lipoproteins. Insulininduced gene (Insig)-1 and Insig-2, two closely related ER membrane proteins, are key negative regulators controlling lipid biosynthesis and uptake through acting on the sterol-regulated maturation of sterol regulatory element-binding proteins (SREBPs) and degradation of HMG-CoA reductase (HMGCR) ${ }^{1-3}$. When cellular sterol level is high, Insigs bind to the SREBP/SREBP cleavage-activating protein (SCAP) complex, blocking its transport from the ER to Golgi and subsequent proteolytic cleavage of the SREBP precursors ${ }^{4}$. As a result, the transcription of lipid biosynthetic genes is downregulated. High concentrations of sterols also promote Insigs and associated ubiquitin ligase gp78 to bind HMGCR, leading to ubiquitylation and proteasomal degradation of $\mathrm{HMGCR}^{5-8}$. Genetic deletion of Insigs resulted in robust increases in mRNA abundance of lipogenic genes and HMGCR protein level, as well as overaccumulation of cholesterol and triglyceride in the liver.

Insig-1 is a short-lived protein in cultured cells. A high concentration of cholesterol promotes SCAP to bind and stabilize Insig-1, whereas cholesterol depletion dissociates the SCAP-Insig1 complex and accelerates Insig-1 ubiquitylation on the K156 and K158 residues by gp78 and degradation ${ }^{9-11}$. Insig-2, albeit sharing about $59 \%$ of sequence identity with Insig-1, is stable in some cultured cells including Chinese hamster ovary (CHO) and human fibroblast SV589 cells ${ }^{12,13}$. However, our previous work showed that Insig-2 was stabilized and accumulated in $g p 78$ deficient mouse liver ${ }^{14}$. The underlying mechanism of tissuespecific Insig-2 stabilization is unknown.

In this study, we generate whole-body $g p 78$ knockout mice and find that the protein level of Insig- 2 is markedly increased in liver, adipose tissue and kidney. However, Insig-2 is surprisingly unaltered in muscle. Using hepatocytes, we demonstrate that Insig- 2 is ubiquitylated on Cys 215 by gp78. On the other hand, oxidization of Cys215 in myotubes by reactive oxygen species (ROS) outcompetes ubiquitylation and protectes Insig-2 from degradation, which prevents muscle cells from lipid overaccumulation. Intriguingly, the $\mathrm{YEC}_{215} \mathrm{~K}$ tetrapeptide of Insig-2 is evolutionarily conserved in amniotes but not in amphibians or fishes, which bear less oxygen and ROS. Together, our study reveals a tissue-specific regulation of Insig-2 stability through oxidation and ubiquitylation on Cys 215 residue and implicates a link between metabolic oxidative state and lipid biosynthesis.

\section{Results}

Insig-2 is unchanged in the muscle of $\boldsymbol{g} \boldsymbol{p} \mathbf{7 8}$-deficient mice. We first generated whole-body $g p 78$ knockout mice $\left(g p 78^{-/-}\right)$by crossing mice with loxP sites flanking the exon 5 and 8 of $g p 78$ $\left(g p 78^{f / f}\right)$ with those expressing Cre recombinase under the control of a cytomegalovirus (CMV) promoter. The $g p 78^{-1-}$ mice were viable and appeared normal. We then compared the Insig-2 protein levels in various tissues between $g p 78^{-/-}$mice and their wild-type (WT) littermates. Ablation of $g p 78$ increased Insig-2 protein in the liver, white adipose tissue and kidney (Fig. 1a). Surprisingly, Insig- 2 protein amount remained unaltered in the skeletal muscle of $g p 78^{-1-}$ mice compared with that of WT controls (Fig. 1a). But the Insig-2 protein was elevated in the heart of $g p 78$-deficient mice (Supplementary Fig. 1a). Since Insig2 negatively regulates SREBP processing ${ }^{1}$, we next analyzed the expression of an array of SREBP target genes in the liver and skeletal muscle by quantitative polymerase chain reaction (qPCR). Except for Insig-2, the mRNA abundance of Srebp-1a,
Srebp-1c, Srebp-2, Insig-1, as well as the genes involved in cholesterol synthesis (Hmgcr and farnesyl diphosphate synthase $[F d s]$ ) and fatty acid and triglyceride (TG) synthesis (fatty acid synthase [Fas], acetyl-CoA carboxylase [Acc], stearoyl-CoA desaturase-1 [Scd-1], glycerol-3-phosphate acyltransferase [Gpat]) were all significantly downregulated in the liver of $g p 78^{-1-}$ mice (Fig. 1b). By contrast, the mRNA levels of all examined genes were similar in the skeletal muscle of $g p 78^{-/-}$ mice and WT controls (Fig. 1c).

Cys215 of Insig-2 is ubiquitynated by gp78 in hepatic cells. It is known that gp78 promotes ubiquitylation and degradation of Insig-1 (ref. ${ }^{10}$ ). To investigate whether gp78 is required for Insig2 degradation, we expressed Myc-tagged Insig- 2 in control and gp78-knockdown CRL1601 cells, and performed a cycloheximide (CHX) chase assay. A rapid degradation of Insig-2 was observed in control RNAi cells, with a half-life of $\sim 3.5 \mathrm{~h}$ (Fig. 2a, b). However, about $80 \%$ of Insig- 2 protein remained stable even after $5 \mathrm{~h}$ of CHX treatment upon $g p 78$ deficiency (Fig. 2a, b).

Human Insig-2 is $59 \%$ identical to Insig-1 and proteasomal degradation of Insig-1 requires ubiquitylation of Lys156 and Lys158

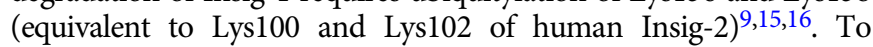
investigate whether the Lys100 and Lys102 residues are responsible for gp78-mediated degradation of Insig-2, we mutated both lysines to arginines (K100R/K102R) and found the mutants degraded as fast as the WT one (Fig. 2c, d). In fact, substituting all cytoplasmic lysines for arginines (K0) did not affect Insig-2 degradation as revealed by $\mathrm{CHX}$ chase analysis (Fig. 2c, d). Because ubiquitin can also be covalently attached to the $\mathrm{SH}$ moiety of cysteine residues ${ }^{17,18}$, we next replaced the cysteine residues on the cytoplasmic side with alanines (Fig. 2c). Insig-2 carrying all three cysteine mutations $(\mathrm{C} 0)$ remained steady at $5 \mathrm{~h}$ of $\mathrm{CHX}$ treatment (Fig. 2e). In particular, the $\mathrm{C} 215 \mathrm{~A}$ mutation but not other single cysteine mutations (C14A and C90A) effectively prevented Insig-2 degradation (Fig. 2e). Together, these results suggest that Cys215 is required for gp78-mediated degradation of Insig-2 in CRL1601 cells.

To exclude the possibility that the $\mathrm{C} 215 \mathrm{~A}$ mutation impairs the interaction between Insig- 2 and gp78, we immunoprecipitated transfected WT Insig-2 and Insig-2 (C215A) proteins using the anti-Myc coupled agarose. As shown in Supplementary Fig. 1b, comparable amounts of gp78 were detected co-immunoprecipitated with WT and mutant Insig-2 proteins. To assess whether the C215A mutation affects Insig-2 function, we examined Insigmediated degradation of the overexpressed HMGCR protein. Both WT and C215A mutant supported sterol-regulated degradation of HMGCR (Supplementary Fig. 1c). Together, these results suggest that the C215A mutation neither alters Insig-2 binding to gp78 nor impairs Insig-2 function in mediating HMGCR degradation.

We next examined whether Insig- 2 could be ubiquitylated by gp78. The Insig-2 protein was immunoprecipitated from control or $g p 78$-knockdown cells in the presence of proteasome inhibitor MG132 followed by immunoblotting analysis with the antiubiquitin antibody. Compared with the high-molecular-weight smear in control cells, substantially reduced ubiquitylation was detected upon $g p 78$ knockdown (Fig. 2f). It has been known that UBE2G2 is the E2 cooperating with gp78 ${ }^{19}$. Knockdown of UBE2G2 dramatically decreased the Insig-2 ubiquitylation (Supplementary Fig. 1d, e). To exclude the possibility that ubiquitylation may take place on Insig-2-associated proteins, we pulled down WT and C215A forms of Insig-2 in the denaturing conditions that disrupt all protein interactions. The ubiquitylation of WT but not mutant Insig-2 was detected (Fig. 2g). Next, we compared the ubiquitylation level of Insig-2 in the absence or presence of DL-Dithiothreitol (DTT) and beta-mercaptoethanol (BME). These reducing agents can disrupt the thioester bond 
a

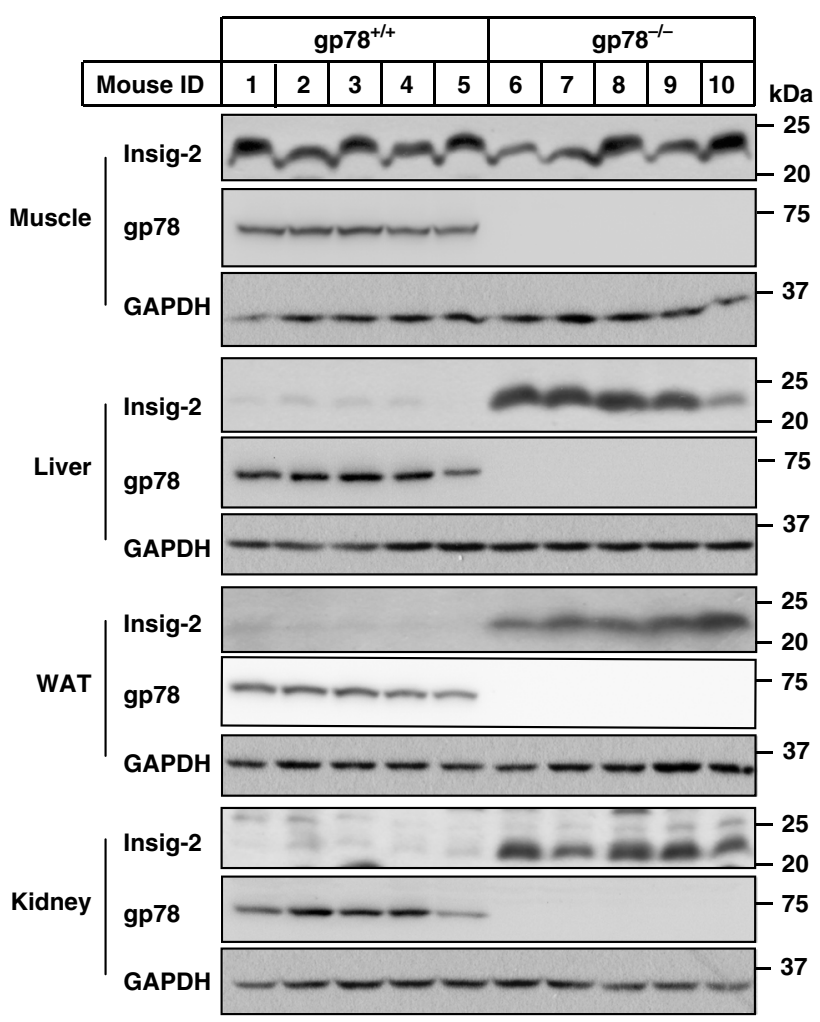

b
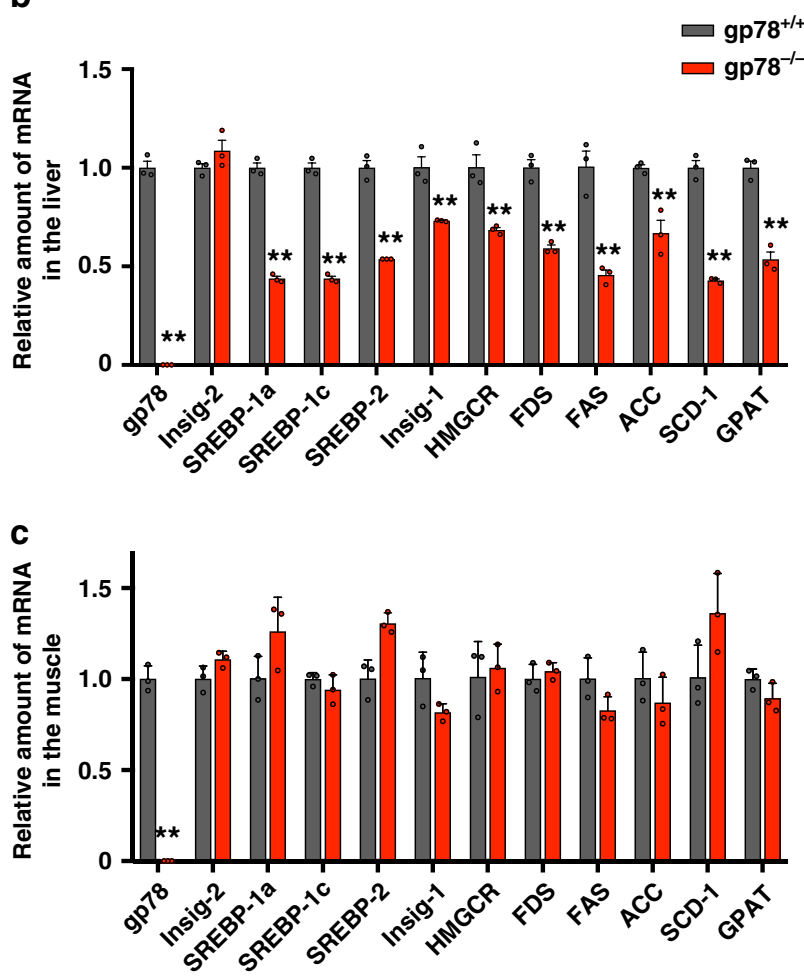

Fig. 1 Ablation of gp78 increases Insig-2 protein level in the liver, WAT and kidney but not in the muscle. Male gp78 ${ }^{-/-}$mice and their wild-type (WT) littermates $\left(\mathrm{gp} 78^{+/+}\right)(8$-week-old, $n=5$ per group) were sacrificed and harvested for immunoblotting (a) and RT-qPCR (b, c) analysis. a Expression of indicated proteins in different tissues. b, c The mRNA levels of indicated genes in the liver (b) and muscle (c). Data are presented as mean $\pm s . d$. ${ }^{\star \star} p<0.01$, unpaired two-tailed Student's $t$ test.

between ubiquitin and cysteine residues, producing unubiquitylated proteins with smaller molecular mass ${ }^{18}$. Indeed, addition of DTT and BME apparently downshifted the transfected Insig-2 protein to its original molecular weight of $\sim 33 \mathrm{kDa}$ (Fig. 2h). In addition, we treated the Insig-2 IP samples with DTT/BME and hydroxylamine. DTT/BME and hydroxylamine could down-shift the ubiquitin signal, suggesting that they broke the thioester bond between cysteine and ubiquitin molecules (Supplementary Fig. 1f). Together, these results demonstrate that Insig-2 is ubiquitylated on Cys 215 by gp78.

gp78 promotes Insig-2 degradation in undifferentiated myoblasts. The interesting observation that $g p 78$ deficiency failed to elevate Insig-2 protein levels in mouse skeletal muscle (Fig. 1a) prompted us to investigate whether gp78 facilitates Insig-2 degradation in $\mathrm{C} 2 \mathrm{C} 12$, an immortalized skeletal myoblast cell line derived from mouse muscle ${ }^{20}$. Consistent with the previous findings ${ }^{21,22}, \mathrm{C} 2 \mathrm{C} 12$ cells remained undifferentiated in normal growth medium (containing 10\% FBS) with fibroblast-like appearance and single rounded nucleus per cell (Fig. 3a). Under low serum ( $2 \%$ horse serum (HS)) conditions, however, C2C12 cells were induced to differentiate and fuse into elongated, multinucleated myotubes over time (Fig. 3a). Meanwhile, we detected drastically increased expression of differentiation markers including $M r f 4, M y o G, M y H C-2 A, M y H C-1, M y H C-2 B$, and $\mathrm{MyHC}-2 \times$ (Fig. 3b). These results validate our myoblast-tomyotube differentiation system.

We then generated two independent lines of $g p 78$-deficient C2C12 cells (gp78-KO \#1 and \#2) using the CRISPR/Cas9 techniques and induced differentiation by supplementing cells with $2 \%$ HS. The CHX chase assay was performed to compare the half-life of transfected Insig-2 in undifferentiated and differentiated C2C12 cells. Similar to the observations in CRL1601 cells (Fig. 2a, lanes 1-3), Insig-2 was barely detectable at $5 \mathrm{~h}$ of $\mathrm{CHX}$ treatment in undifferentiated WT myoblasts (Fig. 3c, lanes 1-3). This Insig-2 degradation, however, was completely abrogated upon $g p 78$ deficiency (Fig. 3c, lanes 4-9). Insig-2 was very stable in both WT and $g p 78 \mathrm{KO} \mathrm{C} 2 \mathrm{C} 12$ cells $6 \mathrm{~d}$ after differentiation (Fig. 3d). The C215A mutation protected Insig-2 from degradation in the undifferentiated cells, with no obvious changes in the stability of WT and mutant Insig-2 proteins in the differentiated myotubes (Fig. 3e). Further, Insig-2 ubiquitylation was substantially reduced following differentiation (Fig. 3f). The immunoprecipitation (IP) assay revealed equal amounts of gp78 interacting with Insig-2 in both differentiated and undifferentiated cells (Supplementary Fig. 1g), suggesting that the binding of gp78 to Insig-2 in $\mathrm{C} 2 \mathrm{C} 12$ cells is constitutive and independent of the differentiation state. We therefore concluded that gp78 accounts for ubiquitylation and degradation of Insig- 2 in the undifferentiated but not differentiated $\mathrm{C} 2 \mathrm{C} 12$ cells. These findings in the cultured cells are consistent with $g p 78^{-1-}$ mouse muscle results (Fig. 1a), both of which suggest that gp78-mediated Insig-2 ubiquitylation and degradation are blunted in the muscle.

Cys215 of Insig-2 is oxidized by ROS. Since Cys 215 is the ubiquitylation site of Insig-2 and cysteines can be readily oxidized, we took advantage of a cysteine sulfenic acid (-SOH) probe DCP-Biol to analyze the redox state of cysteine residues in Insig2 protein (Fig. 4a). DCP-Bio1 recognizes the oxidized form of cysteines in vitro and in vivo ${ }^{23}$. GAPDH can be effectively detected by DCP-Biol (refs. ${ }^{24,25}$ ) and was used as a positive control. The WT form of Insig- 2 was successfully labeled by 

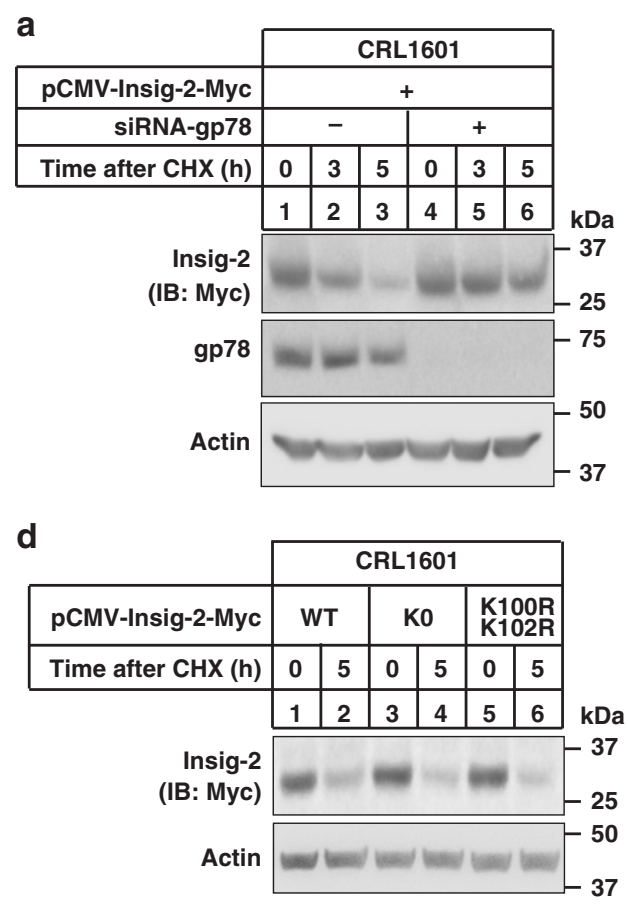
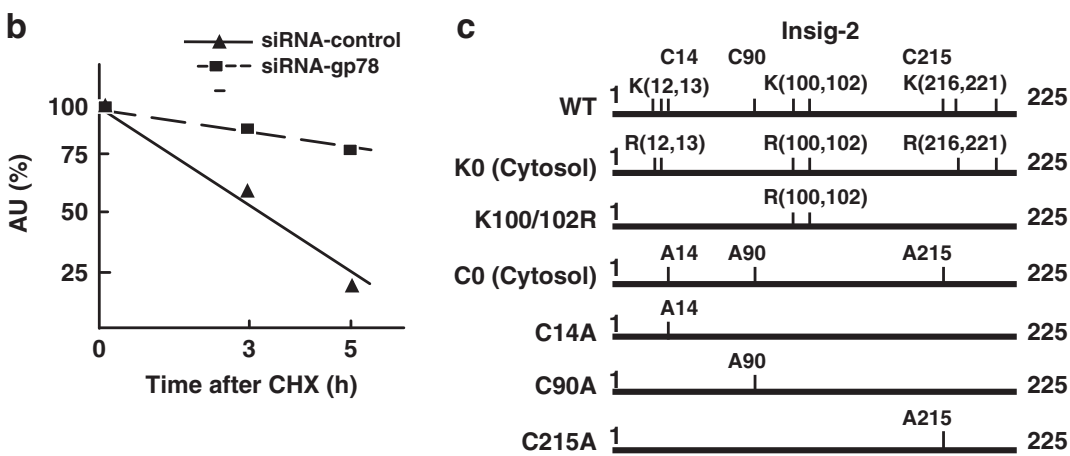

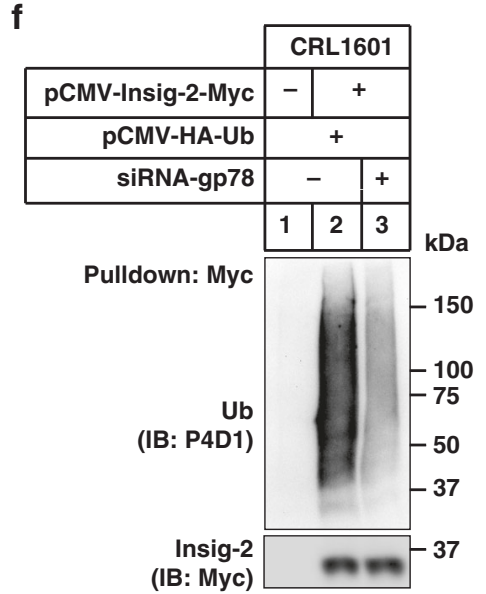

g

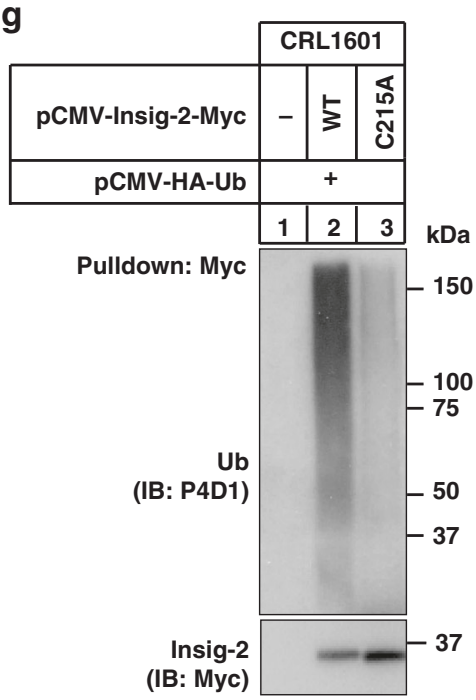

h
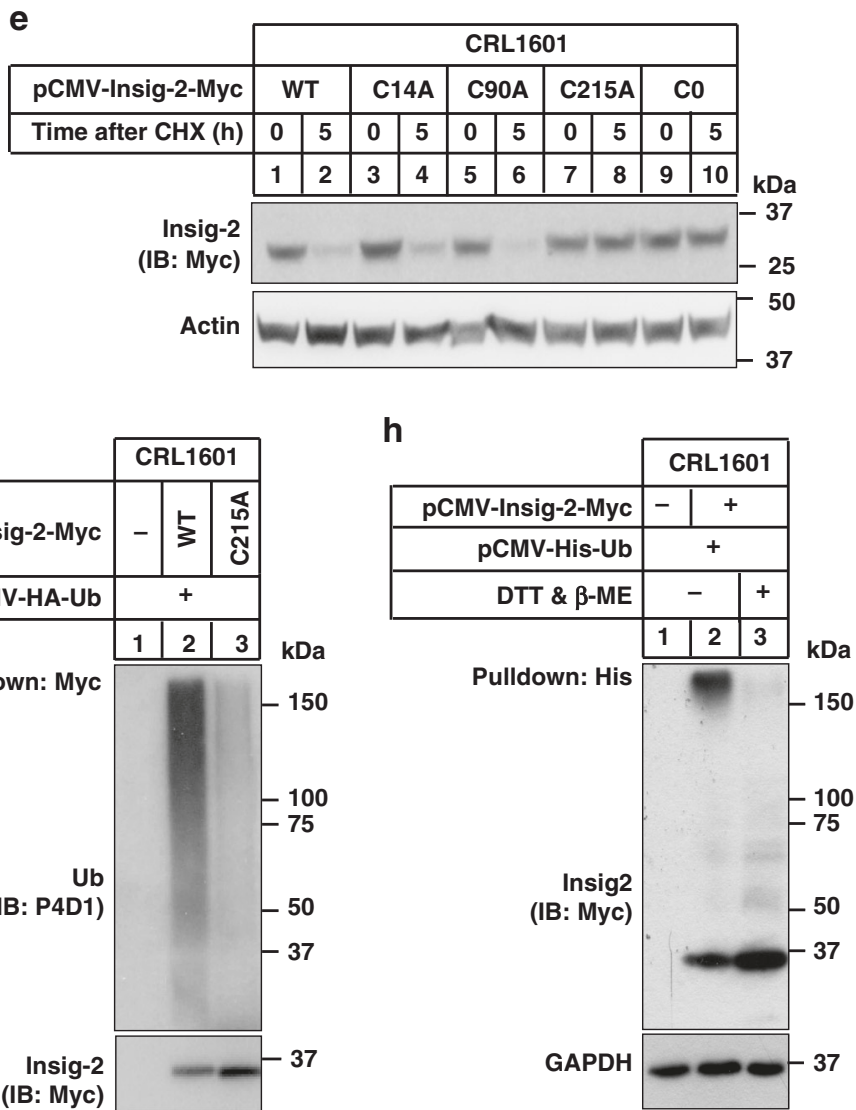

Fig. 2 Ubiquitylation and degradation of Insig-2 requires gp78 and the Cys215 residue. a CRL1601 cells were transfected with the plasmid expressing Myc-tagged Insig-2 and indicated siRNAs. Cells were then treated with $100 \mu \mathrm{M}$ cycloheximide (CHX) for indicated periods and harvested for immunoblotting analysis. b Densitometric analysis ( $n=3$ independent quantification) of Insig-2 levels in (a). AU arbitrary unit. c Schematic of human Insig2 protein and various mutants. d, e CRL1601 cells were transfected with plasmids expressing different Insig-2 variants in (c), and then treated without or with $100 \mu \mathrm{M} \mathrm{CHX}$ for $5 \mathrm{~h}$ followed by immunoblotting analysis. f CRL1601 cells were transfected with indicated plasmids and siRNAs, and treated with 10 $\mu \mathrm{M} \mathrm{MG132}$ for $5 \mathrm{~h}$. Cells were harvested for ubiquitylation assay. $\mathbf{g}$ CRL1601 cells were transfected with indicated plasmids and treated with $10 \mu \mathrm{M}$ MG132 for $5 \mathrm{~h}$. Cells were harvested and lysed in denaturing IP buffer and subjected to ubiquitylation assay. $\mathbf{h}$ CRL1601 cells were transfected with indicated plasmids and treated with $10 \mu \mathrm{M}$ MG132 for $5 \mathrm{~h}$. Cells were harvested, lysed in denaturing IP buffer followed by incubation with the Ni-NTA beads. Precipitates were treated with $(+)$ or without $(-)$ DTT $(75 \mathrm{mM})$ and $\beta-\mathrm{ME}(1.5 \%)$ prior to immunoblotting analysis.

DCP-Bio1 whereas C0 and C215A mutations largely abolished probe recognition (Fig. $4 \mathrm{~b})$. These results suggest that Insig-2 is oxidizable and Cys 215 is the major oxidation site. To determine whether Insig-2 stability is regulated by cellular redox, we transfected cells with Insig-2 followed by treatments with increasing concentrations of hydrogen peroxide $\left(\mathrm{H}_{2} \mathrm{O}_{2}\right)$, an oxidant that can convert the cysteine residues to cysteine sulfenic acids ${ }^{26,27} \cdot \mathrm{H}_{2} \mathrm{O}_{2}$ markedly increased Insig-2 oxidation (Fig. 4c) and protein levels (Fig. 4d). In addition, we also treated cells with three other ROS inducers (palmitic acid, pyocyanin, and menadione) and two scavengers (NAC and Trolox). The ROS levels were significantly increased by palmitic acid (PA), pyocyanin, and menadione, and decreased by NAC and Trolox (Supplementary Fig. 2a). Notably, the three ROS inducers elevated and the two ROS scavengers reduced Insig-2 level (Supplementary Fig. 2b). Importantly, Insig-1 was not labeled by the DCP-Biol probe (Fig. 4e). Sequence alignments showed that both human and mouse Insig-1 proteins lack the $\mathrm{YEC}_{215} \mathrm{~K}$ tetrapeptide as found in Insig-2 (Fig. 4f). These data suggest Insig-2 stabilization is regulated by oxidation at the Cys 215 residue. 
a

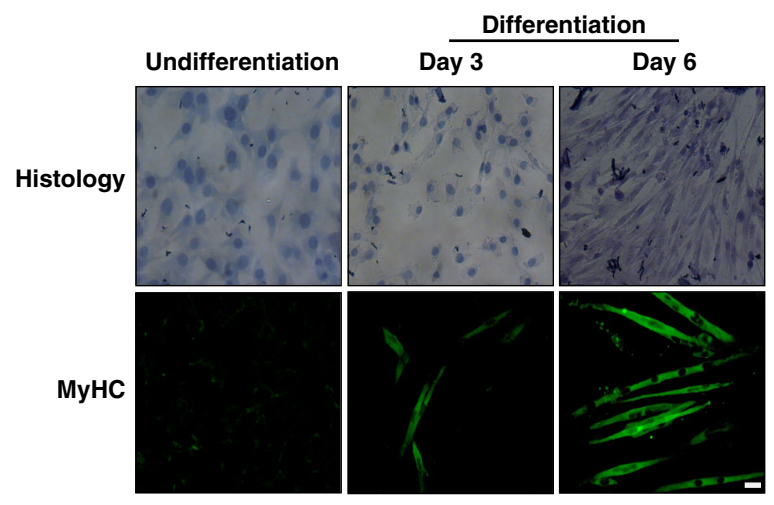

b

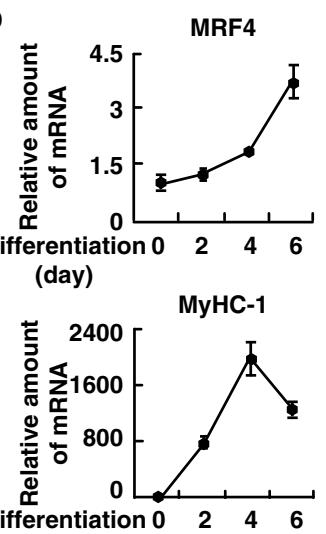

(day)
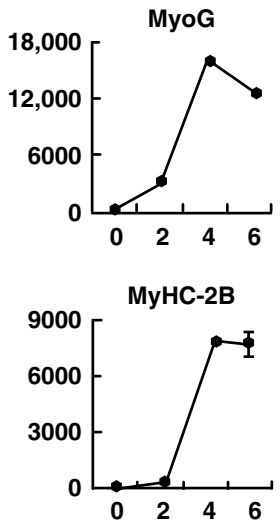

MyHC-2A

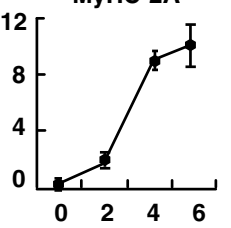

MyHC-2X

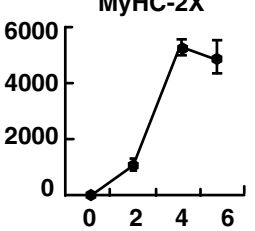

C

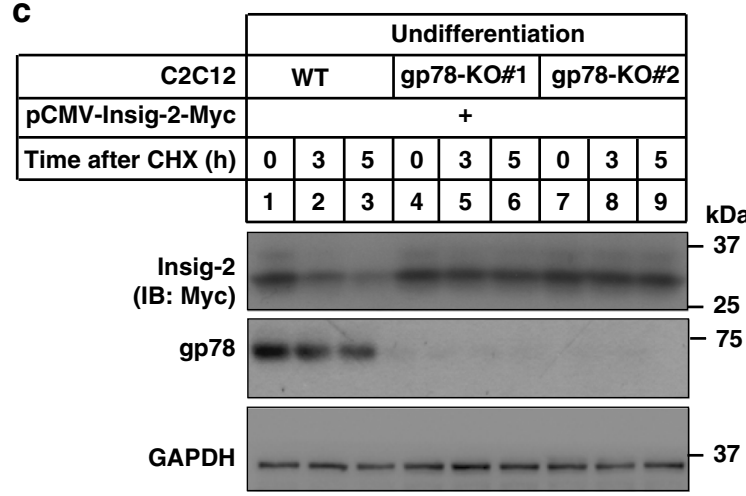

e

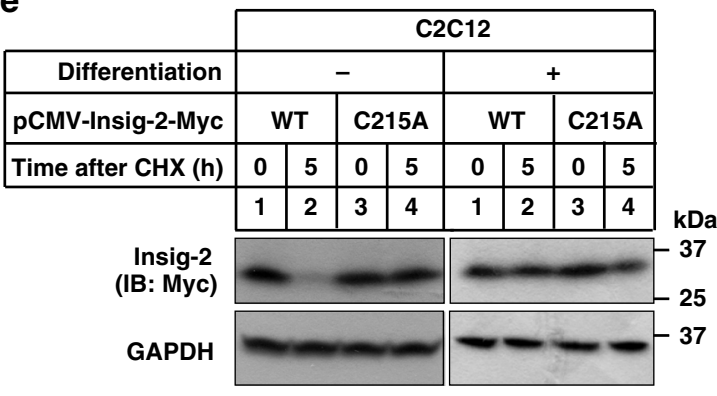

$\longrightarrow-w T$

--E- - gp78-KO\#1

- -- gp78-KO\#1

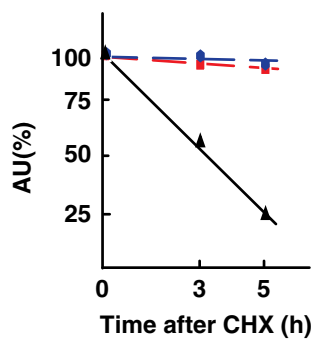

d

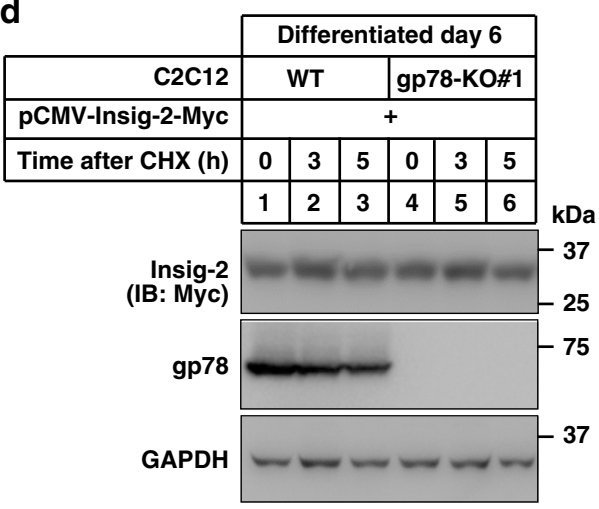

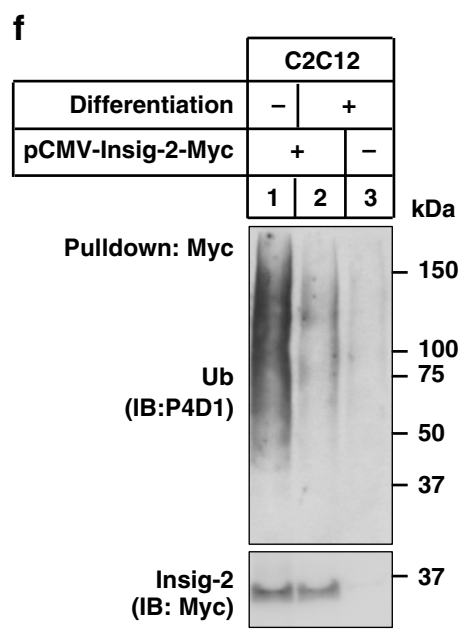

Fig. 3 Gp78 mediates Insig-2 degradation in the undifferentiated myoblasts but not differentiated myotubes. a Histologic and anti-MyHC immunofluorescent images of undifferentiated and differentiated C2C12 cells. Scale bar, $20 \mu \mathrm{m}$. b Quantification of gene expression of several differentiation markers using RT-qPCR ( $n=3$ biologically independent samples per condition, bars in black). Data are presented as mean \pm s.d. $\mathbf{c}$, $\mathbf{d}$ Undifferentiated WT or two lines of gp78-knockout (gp78-KO\#1 and \#2) C2C12 cells (c) and WT or gp78-KO\#1 C2C12 cells at Differentiation Day 6 (d) were transfected with a plasmid expressing Insig-2 and treated with $100 \mu \mathrm{M} \mathrm{CHX}$ for indicated time periods. Cells were harvested for immunoblotting analysis. Densitometric analysis of Insig-2 levels in (c). AU arbitrary unit. e Undifferentiated ( - ) and differentiated (+, Day 6) C2C12 cells were transfected with plasmids expressing WT or C215A mutant forms of Insig-2. Forty-eight hours later, cells were treated without or with $100 \mu \mathrm{M} \mathrm{CHX} \mathrm{for} 5 \mathrm{~h}$. Cells were harvested for immunoblotting analysis. $\mathbf{f}$ Undifferentiated $(-)$ and differentiated $(+) \mathrm{C} 2 \mathrm{C} 12$ cells were transfected with plasmids expressing Myc-tagged Insig-2 and HA-tagged ubiquitin. Forty-eight hours later, cells were treated with $10 \mu \mathrm{M} \mathrm{MG132}$ for $5 \mathrm{~h}$ and harvested for ubiquitylation assay.

Ablation of Insig-2 causes lipid accumulation. We next investigated the mechanism by which Insig-2 is stabilized in the differentiated $\mathrm{C} 2 \mathrm{C} 12$ cells. We measured the ROS level during myoblast differentiation and myotube formation. A gradual increase in the ROS level during $\mathrm{C} 2 \mathrm{C} 12$ differentiation was revealed by dichlorofluorescein (DCF) (Fig. 5a). The ROS level in hepatic CRL1601 cells was in the middle (Supplementary Fig. 2c). Meanwhile, Insig-2 oxidation was increased over the differentiation period (Fig. 5b). This result supported our hypothesis that the skeletal muscle had higher ROS and Insig-2 was easier to be oxidized in skeletal muscle than in other tissues. In addition, we treated the WT C2C12 cells with the ROS 
a

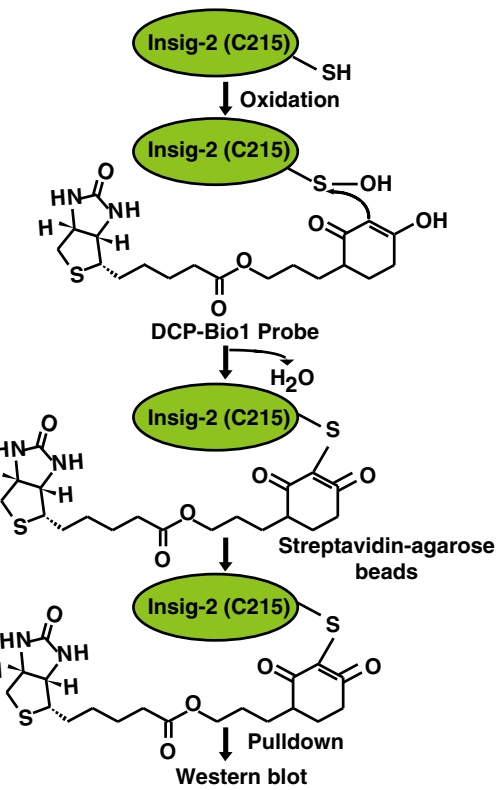

C

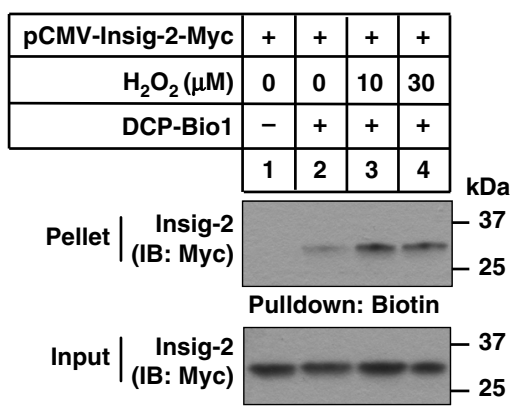

b

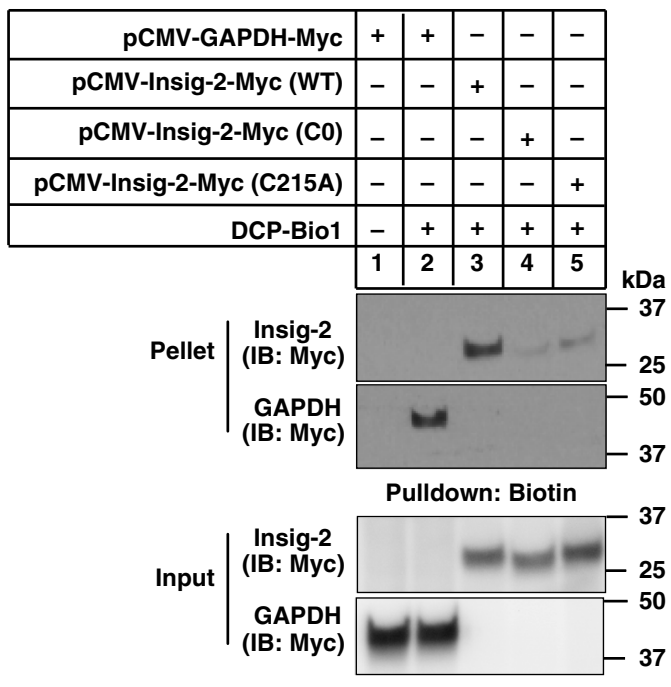

d

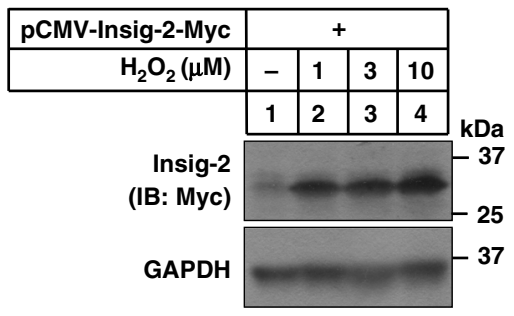

e

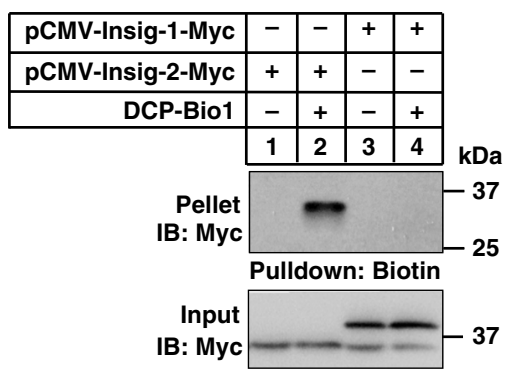

\section{f}

Exon 4

Exon 5

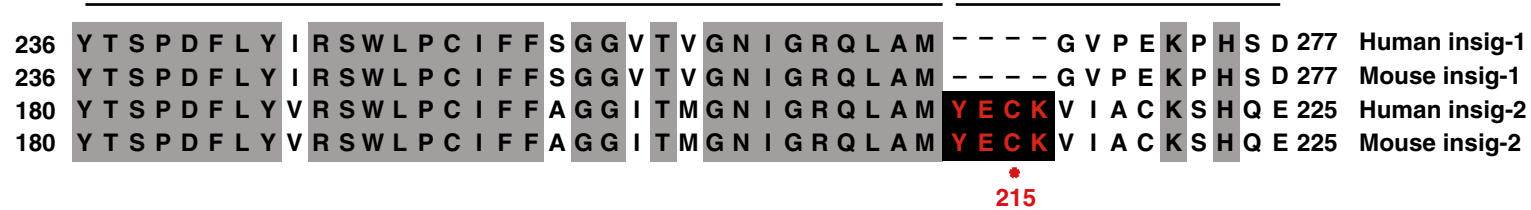

Fig. 4 Cys215 of Insig-2 can be oxidized by ROS. a Procedure of detecting Cys215 oxidation in Insig-2 using the DCP-Bio1 probe. b The CRL1601 cells were transfected with indicated plasmids and harvested for cysteine sulfenic acid detection as described in the Materials and methods. c C2C12 cells were transfected with a plasmid expressing Insig-2 and then treated with $\mathrm{H}_{2} \mathrm{O}_{2}$ at indicated concentrations for 10 min. The cells were then harvested for cysteine sulfenic acid detection. $\mathbf{d}$ C2C12 cells were transfected with a plasmid expressing Insig-2 and then treated without (-) or with $10 \mu \mathrm{M} \mathrm{H} \mathrm{H}_{2} \mathrm{O}_{2}$ for $5 \mathrm{~h}$. Cells were harvested for immunoblotting analysis. e CRL1601 cells were transfected with plasmids expressing Insig-1 or Insig-2 as indicated and then harvested for cysteine sulfenic acid detection. $\mathbf{f}$ Sequence alignment of human and mouse Insig-1 and Insig-2 proteins. Conserved sequences are highlighted in shadow. The YECK tetrapeptide (in red) is exclusively found in Insig-2 but not Insig-1 proteins. Red dot denotes the ubiquitylation site.

scavenger NAC during differentiation. NAC did not affect the cell differentiation, as $\mathrm{MyHC}$ expression pattern was similar between control and NAC treatment. But the Insig-2 protein level in NAC-treated cells was much lower than in control cells (Fig. 5c). These results, together with those in Fig. 4, demonstrate an elevation in the cellular ROS level during muscle cell differentiation, which may lead to Insig-2 oxidation that competes with ubiquitylation-mediated degradation of the protein at Cys215.

To investigate the function of Insig-2 during $\mathrm{C} 2 \mathrm{C} 12$ differentiation, we generated Insig- $2 \mathrm{KO}$ cells using the CRISPR/Cas9 techniques. The expression pattern of $\mathrm{MyHC}$ (Fig. 5d) were similar between WT and Insig-2 KO cells during differentiation, indicating that Insig-2 is not required for myoblast differentiation. The protein level of Insig-2 was increased in WT cells over time (Fig. 5d), supporting the notion that differentiation elevates Insig-2 oxidation and thus prevents its ubiquitylation and degradation. Ablation of Insig-2 significantly elevated the mRNA abundance of Acs, Srebp-1c, Fas, Scd-1, and glucokinase on differentiation day 6 (Fig. 5e), suggestive of enhanced lipid biosynthesis. The expression levels of Adiponectin were similar in WT and Insig-2 KO cells, indicating that Insig-2 deficiency does not interefere adipocyte differentiation (Supplementary Fig. 2d). Consistently, differentiated WT and Insig-2 KO cells exhibited much more Oil Red $\mathrm{O}$ staining compared with undifferentiated counterparts, and ablation of Insig-2 exacerbated the phenotype (Fig. 5f, g). In addition, we isolated satellite cells from WT and Insig-2 KO mice. The cells were induced to muscle cells with $2 \%$ HS for 2 days. The Insig-2-deficient primary muscle cells 


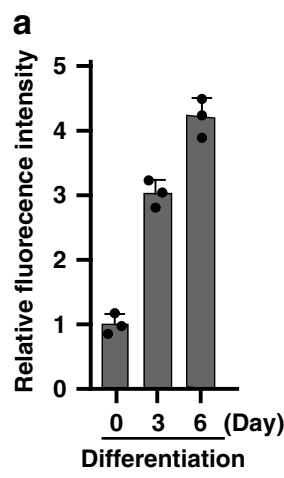

C

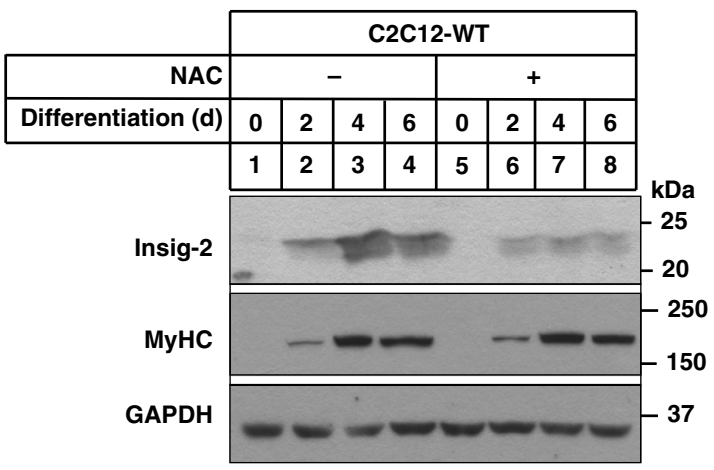

b

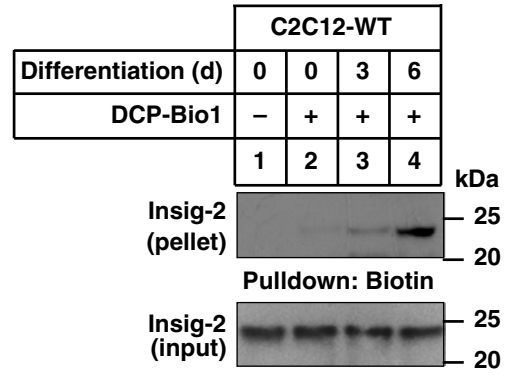

d
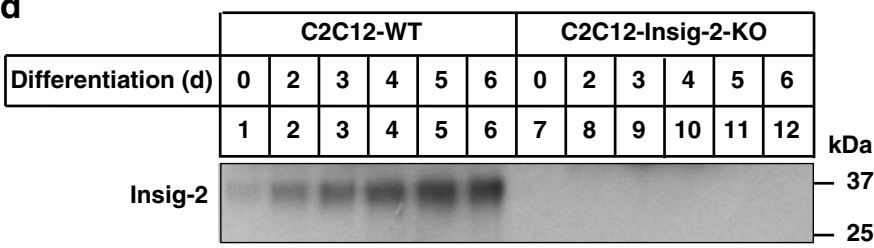

MyHC

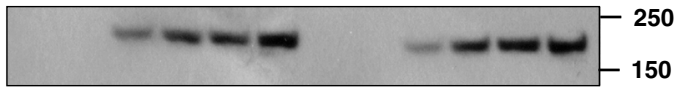

GAPDH
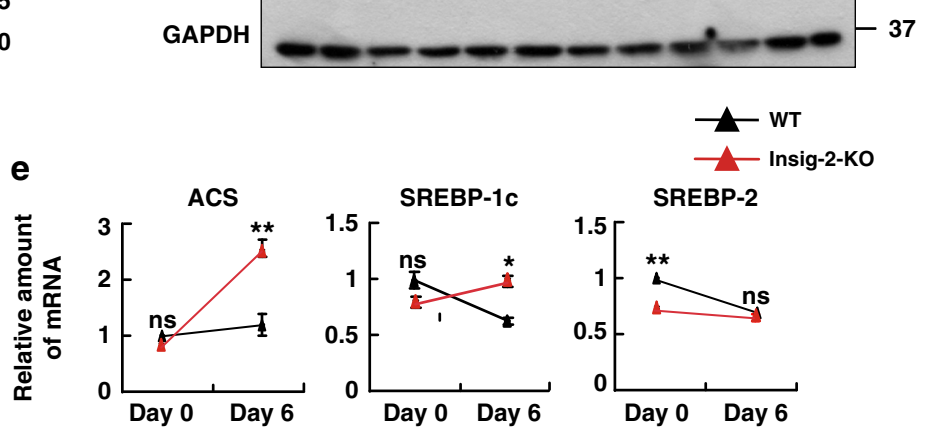

SREBP-2

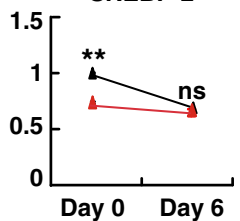

FAS
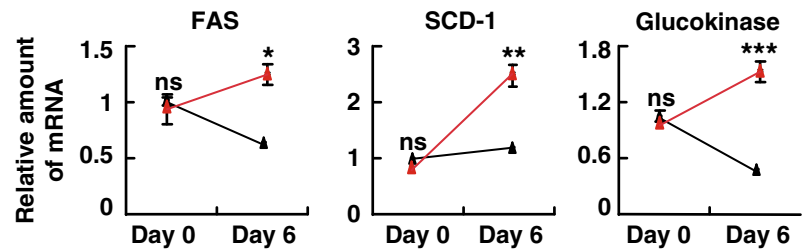

$\mathbf{f}$

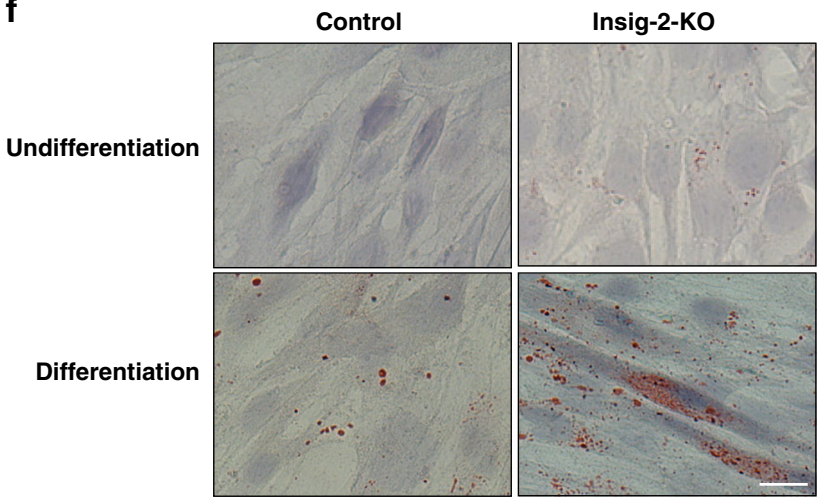

g

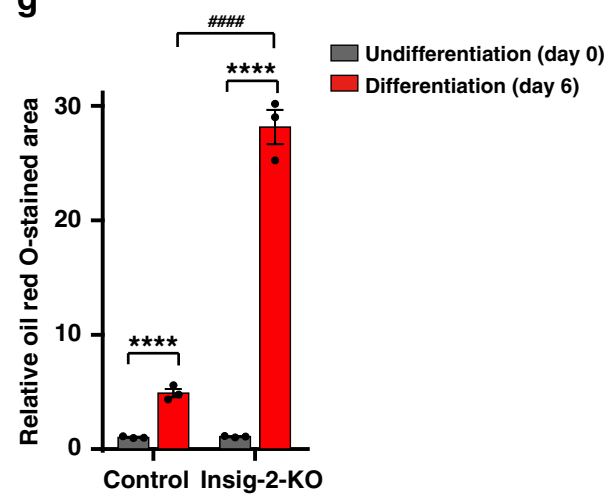

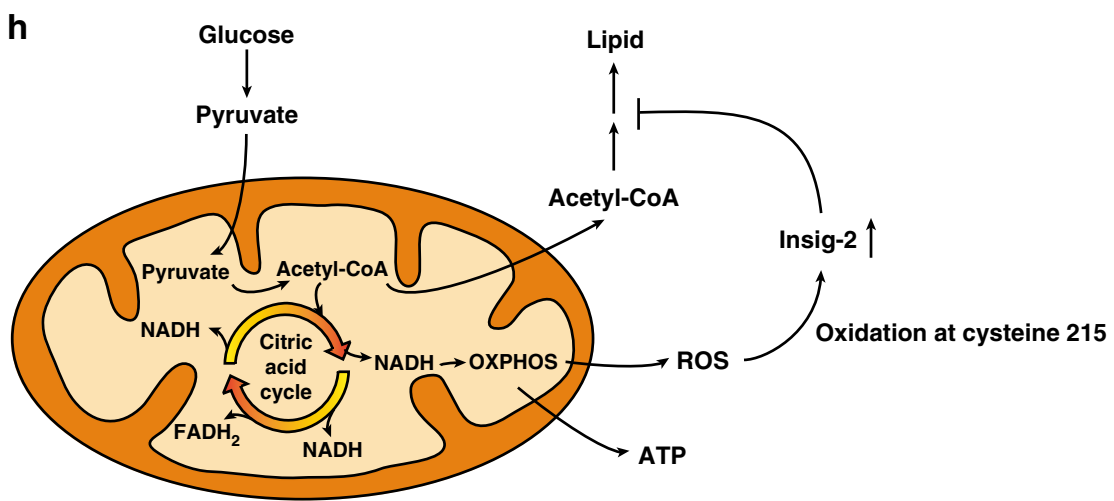

exhibited much more Oil Red O staining compared with WT cells (Supplementary Fig. 2e).

The skeleton muscle is the organ that consumes the majority of glucose in blood. In theory, glucose can be used to generate ATP through glycolysis, TCA cycle, and oxidative phosphorylation with ROS produced as side products. Acetyl-CoA derived from glucose can also be used to synthesize lipids through lipogenesis. The oxidation of C215 by ROS stabilizes Insig-2, thus blocking the SREBP pathway and lipid biosynthesis and helping to channel the carbon flux mainly towards ATP generation. 
Fig. 5 Depletion of Insig-2 increases lipid accumulation in differentiated $\mathbf{C 2 C 1 2}$ cells. a The ROS levels of C2C12 cells during differentiation as measured by dichlorofluorescein (DCF). Data are presented as mean \pm s.d. ( $n=3$ independent experiments). $\mathbf{b}$ C2C12 cells were harvested for cysteine sulfenic acid detection at indicated differentiation days. c WT C2C12 cells treated without or with NAC at indicated differentiation days were harvested for immunoblotting analysis. d WT and Insig-2-KO C2C12 cells at indicated differentiation days were harvested for immunoblotting analysis. e The mRNA levels of indicated genes in the undifferentiated and differentiated $\mathrm{C} 2 \mathrm{C} 12$ cells ( $n=2$ biologically independent samples per condition, bars in black). Data are presented as mean \pm s.d. ${ }^{\star} p<0.05,{ }^{* \star} p<0.01,{ }^{\star *} p<0.001$. ns not significant, two-way ANOVA followed by Bonferroni's post hoc analysis. $\mathbf{f}$ Oil Red $O$ staining, Scale bar, $20 \mu \mathrm{m}$. $\mathbf{g}$ Quantification of the lipid droplets of the cells in ( $\mathbf{f}$ ) ( $n=3$ biologically independent experiments). Data are presented as mean \pm s.d. Asterisks indicate the differences of oil red O staining in WT (bars in black) or Insig-2-KO C2C12 cells (bars in red) between Differentiation Day 0 (undifferentiated) and $6,{ }^{\star \star \star *} p<0.0001$; hash symbols indicate the differences of oil red O staining in WT and Insig-2-KO C2C12 cells at Differentiation Day 6, \#\#\# $p<0.0001$, two-way ANOVA followed by Bonferroni's post hoc analysis. $\mathbf{h}$ Schematic showing glucose metabolism in the skeleton muscle. The oxidation of C215 by ROS generated as side products during oxidative phosphorylation (OXPHOS) process stabilizes Insig-2, inhibiting lipid biosynthesis by blocking the SREBP pathway and helping to channel the carbon flux mainly to ATP generation.

Evolutionary conservation of Insig- 2 in amniotes. To gain a broader picture of Insig-2 evolution, we collected all available vertebrate Insig genes (Supplementary Data set 1). All accessions of genes and genomes are listed in Supplementary Table 1. We obtained complete coding sequences of Insig-2 from 93 species of vertebrates in 5 major groups (mammals, birds, reptiles, amphibians, and fishes) (Fig. 6). By aligning deduced amino acid sequences of these genes, we found a highly conserved YECK tetrapeptide sequence in Insig-2 proteins from all examined reptiles, birds, and mammals (Fig. 6), including secondarily aquatic cetaceans who breathe air as their terrestrial ancestors $\operatorname{did}^{28-30}$. By contrast, most aquatic fishes and amphibians do not have the cysteine residue in such a tetrapeptide (Fig. 6). Based on the principle of parsimony, we speculate that all examined reptiles, birds and mammals have inherited the cysteine residue from their common ancestor of vertebrates, but most aquatic fishes and amphibians do not require such conservation of this residue.

We next examined whether differential selective pressures have acted on the Insig-2 gene between the two vertebrate groups. By using a likelihood method, we estimated $\omega$, the ratio of nonsyonymous to synonymous substitution rates, as a measure of evolutionary force. The $\omega$ values of less than, equal to, and more than 1 represent purifying selection, neutral evolution, and positive selection, respectively ${ }^{31}$. First, we assumed a same $\omega$ for the branches connecting all 93 vertebrates and estimated $\omega$ to be 0.053 (Model A in Supplementary Table 2). We tested whether a model that allows variable $\omega$ for each branch (Model B in Supplementary Table 2; see also Supplementary Fig. 3) fits the data significantly better than Model A. We found that Model B did have a significant better fit than Model A $(P=0.008$, Supplementary Table 2), suggesting that heterogeneous selective pressures must have occurred along specific lineages of vertebrates. Second, we tested whether $\omega$ is on average lower or higher in amniotes than in aquatic fishes/amphibians. Given that secondarily aquatic vertebrates breathe air as their terrestrial ancestors did, we here treated these animals as amniotes. The two-ratio model (model B in Supplementary Table 2) assumes two $\omega$ values: branches in red have $\omega_{1}$ and branches in black have $\omega_{0}$ (Fig. 6), whereas the oneratio model only estimates one uniform $\omega$. Both $\omega_{0}(0.058)$ and $\omega_{1}$ (0.046) estimated by the two-ratio model were smaller than 1 (Supplementary Table 2), indicative of purifying selection. The model B was not significantly different from the model A $(P=$ 0.062 , Supplementary Table 2), suggesting that two groups of vertebrates have a similar $\omega$ value. Third, we tested the possibility of differential selective pressures between two groups of vertebrates by implementing Clade Model $\mathrm{C}(\mathrm{CmC})$ that includes 3 site classes. Classes $0\left(\omega_{0}\right)$ and $1\left(\omega_{1}\right)$ indicate purifying selection and neutral evolution, respectively, and are assumed to be shared between two groups, whereas the third site class is allowed to differ $\left(\omega_{\mathrm{f}} \neq \omega_{2}\right)$ between the focal clade (amniotes, $\omega_{\mathrm{f}}$ ) and the background (aquatic fishes/amphibians, $\left.\omega_{2}\right)^{31}$. After comparing with its null model (M2a_rel) that is same to CmC but assumes $\omega_{\mathrm{f}}=\omega_{2}$ (ref. ${ }^{32}$ ), the difference between two models is statistically significant $(P=0.001$, Supplementary Table 2), suggesting that the $\mathrm{CmC}$ model fits the data significantly better than its null model. A total of $15.3 \%$ amino acid sites of Insig-2 were identified to be under differential selection between amniotes and aquatic fishes/amphibians, with a lower $\omega$ value in the former $\left(\omega_{\mathrm{f}}=0.169\right)$ than the latter $\left(\omega_{2}=0.283\right)$. The greater $\omega$ value suggests that Insig-2 has been relaxed in aquatic vertebrates. Thus, functional divergence of Insig-2 between amniotes and aquatic fishes/amphibians may have been shaped by differential selective pressures.

We next tested whether Insig-2 proteins containing the YECK tetrapeptide sequence are functionally conserved among amniotes. As shown earlier (Fig. 4), human Insig-2 protein is stabilized by oxidation on Cys215. To examine whether Insig-2 with YECK from other species can be regulated by ROS, we synthesized the Insig-2 cDNAs of numerous species and cloned them into expression plasmids for the transfection experiments. We utilized PA to increase cellular ROS level (Fig. 7a) because this condition is more physiologically relevant than $\mathrm{H}_{2} \mathrm{O}_{2}$ treatment. Indeed, high ROS condition increased Insig-2 protein levels of mouse, dog, Baiji dolphin, killer whale, crested ibis, common garter snake, coelacanth, catfish, and whale shark (Fig. 7b). Those without YECK, as in African clawed frog and Nile tilapia, were not altered by ROS (Fig. 7c). Given that the Cys 215 residue in Insig-2 is readily oxidized and that one of the major differences between water and land is oxygen concentration, we then looked into fishes that live in water but have equivalent Cys residues. Nine out of 29 fish species have YECK or YECR (Fig. 6), most of which can either breathe air with a primitive lung (e.g., coelacanth and spotted gar) ${ }^{33}$ or require higher concentration of oxygen in water to survive (e.g., catfish and coast rainbow trout $)^{34,35}$. These exceptions in turn imply an important role of Cys215 oxidation during evolution.

We finally reconstructed the evolutionary history of Insig-1 and Insig- 2 in vertebrates and invertebrates by retrieving genes from GenBank and identifying them from genome or transcriptome assemblies (Supplementary Table 1). Both Insig-1 and Insig-2 were identified in all examined vertebrates. By contrast, only one single gene (named Insig-1/Insig-2) was detected in all examined invertebrates, with the exception of purple sea urchin (Supplementary Fig. 4). The phylogenetic tree showed that vertebrate Insig-1 and Insig-2 formed two separate monophyletic groups deriving from invertebrate Insig-1/Insig-2 (Supplementary Fig. 4), suggesting that the two duplicate genes may have resulted from the whole-genome duplication at the origin of vertebrates ${ }^{36}$.

\section{Discussion}

In this study, we show that Cys215 is the key ubiquitylation site of human Insig-2, and it is also subjected to oxidation by ROS. During myoblast differentiation the cellular ROS level increases, which oxidizes Insig-2 on Cys215 and thus protects the protein 


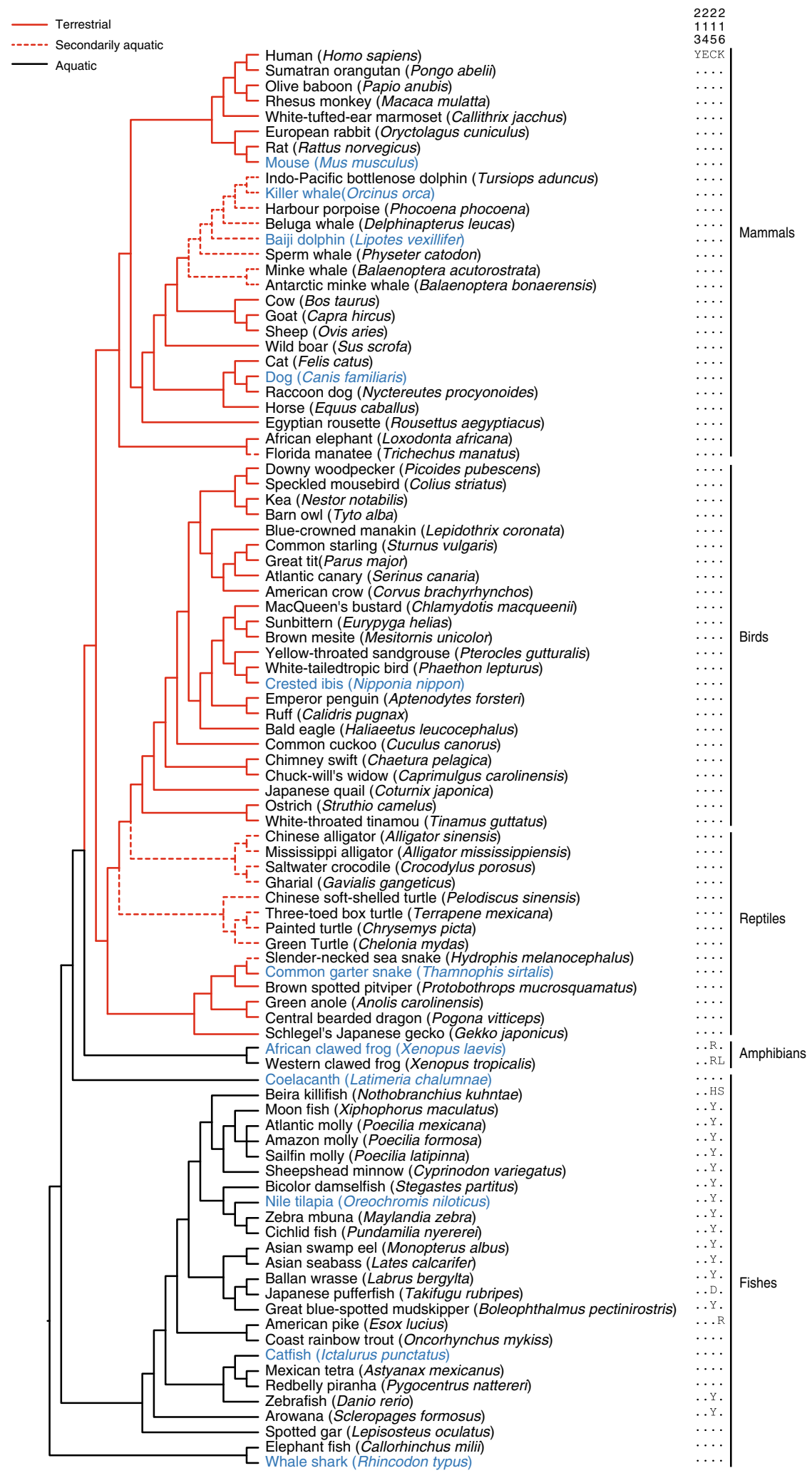

Fig. 6 Sequence conservation of Cys215 of Insig-2 in amniotes during evolution. The tree shows the YECK tetrapeptide sequence (amino acids position of 213-216) of Insig-2 is highly conserved in amniotes ( 93 species in 5 categories, indicated by red lines), including secondarily aquatic cetaceans (indicated by red dashed lines), but not in aquatic amphibians and fishes (indicated by black lines). The species in blue were tested for their Insig- 2 regulation by ROS in Fig. $7 \mathrm{~b}$, c.

from ubiquitylation-mediated degradation in proteasome. This oxidation-ubiquitylation competitive mechanism explains the muscle-specific stabilization of Insig-2 and prevents muscle cells from lipid overaccumulation.
The expression of Insig- 1 and Insig-2 is differentially regulated at both transcriptional and posttranslational levels. Fasting induces high mRNA levels of Insig-2 and low mRNA levels of Insig-1 in the liver ${ }^{37}$. Upon refeeding, insulin downregulates 
a

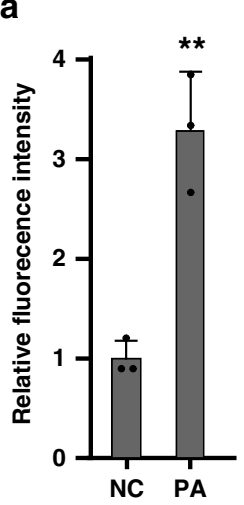

b
C

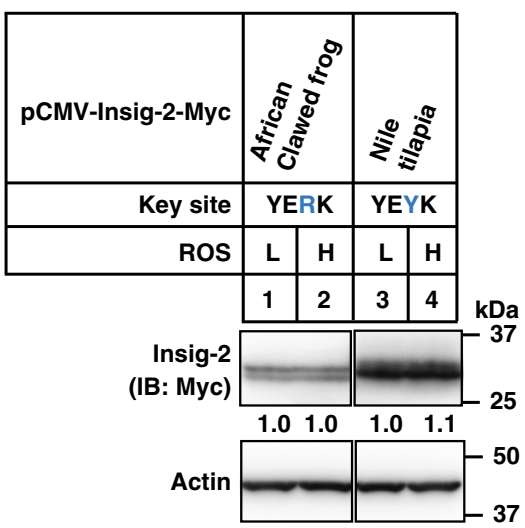

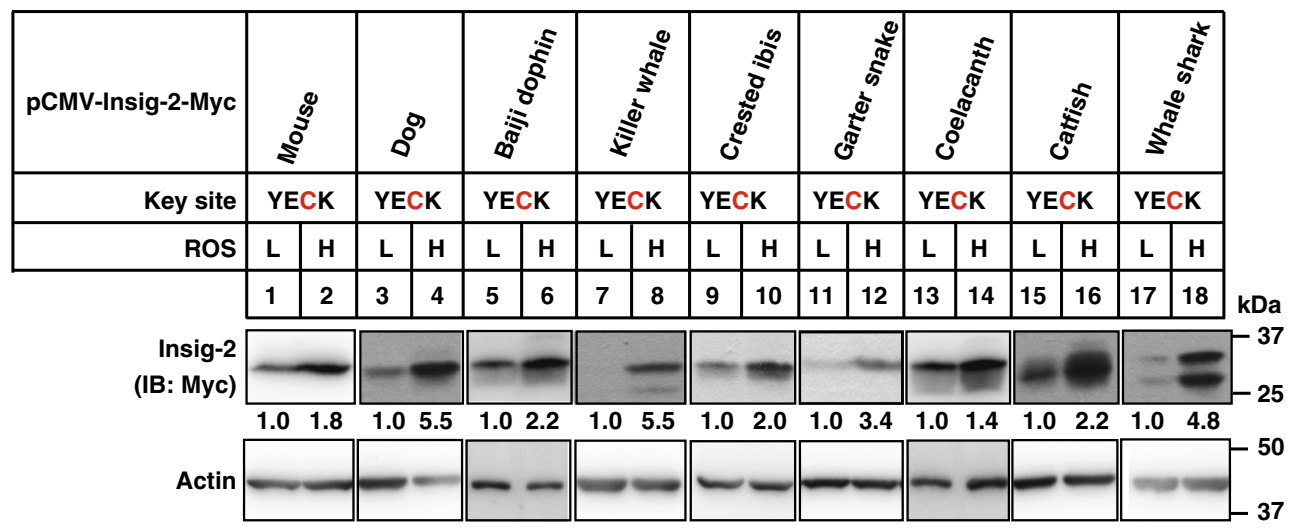

Fig. 7 ROS induces stabilization of Insig-2 protein from representative species of amniotes. a The ROS levels of CRL1601 cells treated without (NC, negative control) or with $200 \mu \mathrm{M}$ palmitic acid (PA) as measured by DCF. Data are presented as mean $\pm \mathrm{s} . \mathrm{d}$. $\left(n=3\right.$ independent experiments). ${ }^{\star \star} p<0.01$, unpaired two-tailed Student's $t$ test. b, c CRL1601 cells were transfected with plasmids expressing Insig- 2 from indicated species and treated without ( $L$, low) or with $200 \mu \mathrm{M}$ PA ( $\mathrm{H}$, high) for $16 \mathrm{~h}$ to induce ROS. Cells were harvested for immunoblotting analysis. Blots were quantified and the intensity of Insig-2 normalized to actin is indicated. Control treatment is defined as 1.

Insig- $2 a$ mRNA, the major transcript of Insig-2, but upregulates the expression of Insig-1 through activating the SREBP-1c pathway. At the protein level, Insig-1 is ubiquitylated and degraded rapidly in response to cholesterol depletion ${ }^{9}$. However, Insig-2 remains stable in cultured fibroblasts and $\mathrm{CHO}$ cells ${ }^{38}$ but is subjected to gp78-mediated ubiquitylation and degradation in mouse liver ${ }^{14}$. We here demonstrate that competitive oxidation and ubiquitylation on the cysteine 215 residue confers the tissuespecific stabilization of Insig-2, hereby reconciling the apparent discrepancy in previous studies. However, the oxidation of endogenous Insig-2 in muscle has not been directly illustrated. We cannot exclude the possibility that Insig- 2 is stabilized by a different mechanism.

Insig-2 was stable in SRD-13A cells unless E214 was mutated to $\mathrm{A}^{39}$. The Insig-2(E214A) was ubiquitinated at K100 and K102 by the E3 gp78. However, the WT Insig-2 was ubiquitinated at C215 if it was not oxidized. The E214A mutation dramatically enhanced the Insig-2-gp78 interaction ${ }^{40}$. But the C215A did not affect Insig-2-gp78 interaction (Supplementary Fig. 1b). In addition, the ubiquitylation sites of Insig-2(WT) and Insig-2 (E214A) were different, it was possible that gp78 bound WT and the E214A mutant Insig-2 in a different way.

The YECK tetrapeptide sequence is exclusively present in Insig2 but not Insig-1. Evolutionary analysis suggests that the two Insig genes may have emerged at the origin of vertebrates through gene duplication (Supplementary Fig. 4). Interestingly, the coding sequence for YECK locates at the exon-intron junction (Fig. 4f), suggesting that alternative splicing may account for the appearance/disappearance of YECK in Insig-2/Insig-1. The Insig-2 proteins in mammals, birds, and reptiles contain the conserved YECK sequence, whereas Insig-2s in most of aquatic fishes and amphibians do not. ROS can stabilize YECK-containing Insig-2 proteins from several distinct species (Fig. 7b), but is ineffective on Insig-2 proteins lacking the YECK sequence (Fig. 7c).

We searched human database in http://myhits.vital-it.ch/cgibin/pattern_search and found about 148 proteins with YECK motif. We randomly chose three membrane proteins to test whether the Cys can affect protein stability. Their half-lives were not affected by the $\mathrm{C}$ to A mutation (Supplementary Fig. 5). These results suggest that YECK is necessary, but not sufficient for the ROS-regulated protein stability.

Why is Insig-2 selectively stabilized in muscle? The skeletal muscle is the major glucose-consuming tissue that takes up approximately $80 \%$ of glucose in the postprandial state ${ }^{41}$. In addition, glucose uptake in exercising skeletal muscle is at least six times more than resting skeletal muscle ${ }^{42}$. Glucose can be used to generate metabolic energy with ROS as side products ${ }^{43}$, as well as to be converted to fatty acids through lipogenesis pathway that is negatively controlled by Insigs. Therefore, our data suggest that the ROS burden may be higher in muscle than the other detected tissues, which is the major reason accounting for the tissueselectivity of Insig-2 stablization. Considering the conservation of the YECK tetrapeptide of Insig-2 in amniotes but not in aquatic animals, we hypothesize that such conservation is not required in aquatic vertebrates due to the low concentration of oxygen. The elevated ROS in muscle induces Insig-2 oxidization at Cys215, 
blocks ubiquitylation and degradation of the protein, which inhibits lipid biosynthesis through attenuating the SREBP pathway and accelerating degradation of HMGCR. This stabilization of Insig-2 by oxidation prevents muscle cells from lipid overaccumulation and could assure proper muscle function that is essential for amniotes during predator-prey interaction. However, for most aquatic vertebrates, the oxygen content in water is much lower and oxidative stress is less ${ }^{44}$, correlating with the absence of the cysteine residue in the corresponding tetrapeptide. As the vertebrates transitioned to terrestrial environments about 380 million years ago when atmospheric $\mathrm{O}_{2}$ was about $25 \%$ and rapidly rose to $>30 \%{ }^{45-49}$ (Supplementary Fig. 6). It will be interesting to investigate whether the ROS-regulated stabilization of Insig-2 plays a role in water-to-land transition.

The oxidation-ubiquitylation competition has been previously reported occurred on acyl-CoA: cholesterol acyltransferase-2 (ACAT-2) protein ${ }^{18}$. The ubiquitylation site of ACAT-2 is also a cysteine residue (Cys277) and can be oxidized by lipid-induced ROS. The oxidation of Cys277 stabilizes ACAT-2, which then converts excess cholesterol and fatty acids to cholesteryl esters so as to reduce lipotoxicity. Here, ROS can competitively block ubiquitylation of Insig-2 on the Cys215 residue via oxidization, stabilizing the protein, and preventing lipid accumulation in muscles. Therefore, competitive oxidation and ubiquitylation on cysteine may be a common mechanism regulating lipid metabolism in response to oxidative stress.

\section{Methods \\ Reagents. We obtained cycloheximide, diethylene triamine pentacetate acid (DTPA), N-ethylmaleimide (NEM), iodoacetamide, catalase, palmitic acid, fatty acid-free bovine serum albumin (BSA), protein inhibitors, $2^{\prime}, 7^{\prime}$-Dichloro- fluorescein diacetate (DCFH-DA) fluorescent dyes, Oil Red O, anti-Myc-coupled agarose beads from Sigma; high capacity NeutrAvidin agarose, mini dialysis devices, 10K MWCO, and ECL from ThermoFisher Scientific. We obtained Ni- NTA agarose beads from Qiagen; MG132 from Calbiochem; M-MLV Reverse Transcriptase from Promega; KOD Hot Start DNA Polymerase from Novagen; Taq polymerase, dNTPs, and X-Gal from TaKaRa; Quick change Site-Directed Muta- genesis kit from Stratagene; Cysteine Sulfenic acid probe DCP-Bio1 from Millipore; hydrogen peroxide $\left(\mathrm{H}_{2} \mathrm{O}_{2}\right)$ from Sinopharm Chemical Reagent Co.,Ltd.; hema- toxylin from Beyotime.}

Primary antibodies. Primary antibodies used for immunoblotting were as follows: mouse monoclonal anti-ubiquitin antibody P4D1 (SC-8017, Santa Cruz, 1:500); mouse monoclonal anti- $\beta$-actin (A1978, Sigma, 1:10,000); rabbit polyclonal antiMyc (06-549, Millipore, 1:1000); rabbit polyclonal anti-Insig-2, rabbit polyclonal anti-gp78, and mouse monoclonal anti-Myc (9E10) were prepared in our laboratory.

Plasmids. The expression plasmids of $5 \times$ Myc-Insig-2, 5x Myc-Insig-1, 5x MycGAPDH, HA-Ub, His $_{6}-\mathrm{Ub}$, and HA-gp78 were constructed by standard molecular cloning techniques. Various Insig-2 site-directed mutants were constructed using the QuikChange site-directed kits and confirmed by sequencing. The Insig-2 cDNAs of representative species were synthesized and cloned into expression plasmid pCMV-5x Myc by Nanjing IDO biotechnology company.

Animals and treatment. The $g p 78^{f / f}$ mice were crossed with cytomegalovirus (CMV) promoter-driven Cre mice (B6.C-Tg(CMV-cre)1Cgn/J, JAX 006054) to generate whole-body $g p 78$ knockout mice $\left(g p 78^{-/}\right)$and crossed with C57BL/6J background for more than ten generations. In addition, Insig $1^{f / f}$ Insig $2^{-1-}$

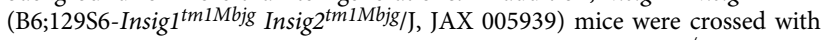
C57BL/6J mice to generate global Insig2 knockout mice (Insig2 ${ }^{-/-}$), which were then backcrossed to the C57BL/6J mice for more than ten generations. Mice were housed under a 12-h light/dark cycle in a pathogen-free, temperature controlled room. Eight-week-old male mice were treated as indicated in the figure legends. All animal experiments were approved and supervised by the Laboratory Animal Ethical Committee of Wuhan University.

Media. Medium A contains Dulbecco's Modified Eagle Medium (DMEM) containing 100 units $\mathrm{mL}^{-1}$ penicillin and $100 \mu \mathrm{g} \mathrm{mL}^{-1}$ streptomycin sulfate. Medium B contains a 1:1 mixture of Ham's F-12 Medium and DMEM containing 100 units $\mathrm{mL}^{-1}$ penicillin and $100 \mu \mathrm{g} \mathrm{mL}^{-1}$ streptomycin sulfate. Lipid-depleting medium was prepared by adding $5 \%$ lipoprotein-deficient serum, $1 \mu \mathrm{M}$ lovastatin and 50 $\mu \mathrm{M}$ mevalonate into each medium.

Cell culture. CHO-7 ( $\mathrm{CHO}$ cell, a clone of $\mathrm{CHO}-\mathrm{K} 1$ cells) was a generous gift from Dr. Joseph Goldstein at UT Southwestern Medical Center, USA. CRL1601 (McArdle RH7777, rat liver cells) and $\mathrm{C} 2 \mathrm{C} 12$ (mouse skeletal muscle cells) were purchased from ATCC. All cells were grown in a monolayer at $37^{\circ} \mathrm{C}$ with $5 \% \mathrm{CO}_{2}$. CRL1601 cells were maintained in medium A supplemented with $10 \%$ fetal bovine serum (FBS). CHO-7 cells were maintained in medium B supplemented with $5 \%$ FBS. C2C12 cells were maintained in medium A supplemented with $10 \%$ FBS. C2C12-sgRNAs-gp78, C2C12-sgRNAs-Insig-2 cells were generated by the CRISPR-Cas9 technology and maintained in medium A supplemented with $10 \%$ FBS. C2C12 cells were induced to differentiate in medium A supplemented with $2 \%$ HS. Muscle stem cells were grown in F10 medium with $20 \%$ FBS, plus IL-1a, IL-13, TNF- $\alpha$, IFN- $\gamma$, and bFGF.

Transfection. C2C12 cells were transfected with indicated plasmids using lipofectamine 3000 transfection reagent (ThermoFisher Scientific). Other cells were transfected using Fugene HD (Promega) according to the manufacturer's instructions.

Measurement of intracellular ROS levels. Intracellular ROS level was determined using oxidation-sensitive DCFH-DA fluorescent dyes. Cells were washed twice with PBS and labeled on the culture plates with DCFH-DA for $30 \mathrm{~min}$ at $37^{\circ}$ $\mathrm{C}$ in serum-free medium. At the end of incubation, culture plates were trypsinized, resuspended in PBS and analyzed using a FACScan flow cytometer $\left(\lambda_{\mathrm{ex}}=488 \mathrm{~nm}\right.$ and $\lambda_{\mathrm{em}} 530 \mathrm{~nm}$ band-pass filter). The mean fluorescence intensity of 10,000 cells was measured in each sample and corrected for autofluorescence from unlabeled cells.

Detection of cysteine sulfenic acid by DCP-Bio1 probe. Cysteine sulfenic acid detection by DCP-Biol probe was performed ${ }^{50}$. The treated cells were washed with PBS and homogenized in DCP-Biol working lysis buffer (1× PBS, $1 \%$ NP40, $1 \%$ sodium deoxycholate, $5 \mathrm{mM}$ EDTA, $5 \mathrm{mM}$ EGTA, $1 \mathrm{mM}$ PMSF, $0.1 \mathrm{mM}$ aprotinin $0.1 \mathrm{mM}$ leupeptin, $10 \mathrm{mM}$ NEM, $10 \mathrm{mM}$ iodoacetamide, $100 \mu \mathrm{M}$ DTPA, and 200 $\mathrm{U} \mathrm{mL}^{-1}$ catalase). Lysates were centrifuged at $12,000 \times g$ for $10 \mathrm{~min}$. Supernatants were collected and incubated with $1 \mathrm{mM}$ DCP-Biol probe for $2 \mathrm{~h}$ on ice. For affinity capture of the labeled proteins, unreacted DCP-Biol was removed using a dialysis device. Dialyzed Samples were precleared with Sepharose CL-4B beads (Sigma), applied to plugged columns containing high-capacity streptavidin-agarose beads (ThermoFisher Scientific) and incubated overnight at $4{ }^{\circ} \mathrm{C}$. Beads were then subjected to a series of stringent washes (at least four column volumes and two washes each) using $1 \%$ sodium dodecyl sulfate (SDS), $4 \mathrm{M}$ urea, $1 \mathrm{M} \mathrm{NaCl}, 10 \mathrm{mM}$ DTT, $100 \mu \mathrm{M}$ ammonium bicarbonate and water. Beads were boiled for $10 \mathrm{~min}$ and samples were analyzed by immunoblotting analysis.

RNA interference. Duplexes of siRNA targeting rat $g p 78$ (5'-CAUGCAGAAUG UCUCUUAAdTdT-3') were synthesized by Genepharma (Shanghai, China). Transfection of siRNA was carried out using lipofectamine RNAiMAX reagent (ThermoFisher Scientific).

Ubiquitylation assays. For ubiquitylation assay in the non-denaturing condition, cells were homogenized in buffer A $(1 \times$ PBS, $1 \%$ NP- $40,1 \%$ sodium deoxycholate and $5 \mathrm{mM}$ EDTA, $5 \mathrm{mM}$ EGTA, $0.1 \mathrm{mM}$ leupeptin) supplemented with protease inhibitors (10 $\mu$ M MG132, $10 \mathrm{mM}$ NEM, and cocktail). Immunoprecipitation was carried out with the anti-Myc agarose beads. For ubiquitylation assay in the denaturing conditions, cells were homogenized in $0.1 \mathrm{~mL}$ buffer A supplemented with protease inhibitors ( $10 \mu \mathrm{M}$ MG132, $10 \mathrm{mM}$ NEM, and cocktail). After sonication and centrifugation, supernatants were collected and mixed with $0.3 \mathrm{~mL} 8 \mathrm{M}$ urea, rotated for $10 \mathrm{~min}$ at room temperature, diluted with buffer A to make final concentration of urea to $2 \mathrm{M}$, and subjected to IP with the anti-Myc agarose. Beads were boiled for $10 \mathrm{~min}$ or incubated with the Myc peptide for $3 \mathrm{~h}$ at $4{ }^{\circ} \mathrm{C}$, and supernatants were analyzed by immunoblotting with indicated antibodies. No DTT or BME was applied unless indicated.

Coimmunoprecipitation. Cells were harvested and lysed in $0.6 \mathrm{~mL}$ of IP buffer ( $1 \times$ PBS, $1 \%$ digitonin, $5 \mathrm{mM}$ EDTA, $5 \mathrm{mM}$ EGTA, and cocktail) followed by centrifugation at $12,000 \times g$ for $10 \mathrm{~min}$ at $4{ }^{\circ} \mathrm{C}$. IP was carried out with indicated antibody-coupled agarose, washed with IP buffers followed by western blotting ${ }^{6}$.

Immunoblotting analysis. Cells were harvested and homogenized in $120 \mu \mathrm{L}$ of RIPA buffer supplemented with protease inhibitors. Protein concentration of whole cell lysates was determined according to the Lowry method (Bio-Rad). Samples were mixed with $4 \times$ SDS loading buffer (150 mM Tris-HCl, pH 6.8, $12 \%$ SDS, 30\% glycerol, $0.02 \%$ bromophenol blue, $6 \%$ BME) and boiled for $10 \mathrm{~min}$. Proteins were resolved by SDS polyacrylamide gel electrophoresis and transferred onto polyvinylidene fluoride membranes. Immunoblots were blocked with 5\% milk in TBST 
containing $0.075 \%$ Tween and probed with primary antibodies overnight at $4{ }^{\circ} \mathrm{C}$. After washing in TBST 3 times, blots were incubated with secondary antibodies for $1 \mathrm{~h}$ at room temperature ${ }^{51}$.

Immunofluorescence. Cells grown on coverslips were fixed with $4 \%$ paraformaldehyde for $30 \mathrm{~min}$ and incubated with $3 \%$ BSA in PBS containing primary antibodies (1:500) for $1 \mathrm{~h}$ at RT. After washing 3 times with PBS, cells were incubated with PBS containing 3\% BSA and corresponding secondary antibodies (1:500) for $1 \mathrm{~h}$ at $\mathrm{RT}^{52}$.

RT (reverse transcription)-qPCR. Total RNA was extracted using Trizol reagents (ThermoFisher Scientific) and subjected to RT with oligo dT followed by qPCR using target-specific primers in the Stratagene Mx30005P Q-PCR Systems. All reactions were prepared in triplicate and the relative amounts of mRNAs were calculated using the comparative CT method. Mouse GAPDH was used as controls. Values show the amount of mRNA relative to control sample, which is arbitrarily defined as 1 . Primer sequences are used as follows:

Mouse Insig-2 (Forward: 5'-CGGAAGATGCTGGAACCTGA-3', Reverse:

5'-TGTGCTCTCCATACGCTCTCC-3');

Mouse $g p 78$ : (Forward: 5'-CTGCCCTGTGGACATCTTTT-3', Reverse: 5'-GAC

CCATCGAAGTGGAAGAA-3');

Mouse SREBP-1a: (Forward: 5'-GGCCGAGATGTGCGAACT-3', Reverse:

5'-TTGTTGATGAGCTGGAGCATGT-3');

Mouse SREBP-1c: (Forward: 5'-GGAGCCATGGATTGCACATT-3', Reverse:

$5^{\prime}$-GGCCCGGGAAGTCACTGT-3')

Mouse SREBP-2: (Forward: 5'-GCGTTCTGGAGACCATGGA-3', Reverse:

5'-ACAAAGTTGCTCTGAAAACAAATCA-3');

Mouse Insig-1: (Forward: 5'-TCACAGTGACTGAGCTTCAGCA-3', Reverse:

5'-TCATCTTCATCACACCCAGGAC-3');

Mouse HMGCR: (Forward: 5'-CTTGTGGAATGCCTTGTGATTG-3', Reverse:

5'-AGCCGAAGCAGCACATGAT-3');

Mouse FDS: (Forward: 5'-ATGGAGATGGGCGAGTTCTTC-3', Reverse:

5'-CCGACCTTTCCCGTCACA-3');

Mouse FAS: (Forward: 5'-GCTGCGGAAACTTCAGGAAAT-3', Reverse:

5'-AGAGACGTGTCACTCCTGGACTT-3');

Mouse ACC: (Forward: 5'-TGACAGACTGATCGCAGAGAAAG-3', Reverse:

5'-TGGAGAGCCCCACACACA-3');

Mouse SCD-1: (Forward: 5'-CCGGAGACCCCTTAGATCGA-3', Reverse:

5'-TAGCCTGTAAAAGATTTCTGCAAACC-3');

Mouse GPAT: (Forward: 5'-CAACACCATCCCCGACATC-3', Reverse:

5'-GTGACCTTCGATTATGCGATCA-3');

Mouse ME: (Forward: 5'-GCCGGCTCTATCCTCCTTTG-3', Reverse: 5'-TTT

GTATGCATCTTGCACAATCTTT-3');

Mouse G6PD: (Forward: 5'-GAACGCAAAGCTGAAGTGAGACT-3', Reverse: 5'-TCATTACGCTTGCACTGTTGGT-3').

Rat UBE2G2: (Forward: 5' -TGGGAGGCATTGATCATGGG-3' ${ }^{\prime}$, Reverse: 5' ${ }^{\prime}$ GC AGGATGGAGATGCACACT-3').

Evolutionary analysis. We collected all available vertebrate Insig-2 genes from the National Center for Biotechnology Information (NCBI), and employed TBLASTN ${ }^{53}$ using the human Insig-2 sequence as query to identify cetacean Insig-2 genes in six cetacean genomes and one reptile genome. Complete coding sequences of Insig-2 from 86 species of vertebrates were downloaded from GenBank (NCBI, www.ncbi. nlm.nih.gov). Insig-2 genes from six species of cetaceans (Indo-Pacific bottlenose dolphin, harbor porpoise, beluga whale, sperm whale, minke whale, and Antarctic minke whale) and one species of reptile (slender-necked sea snake) were identified from their genome assemblies by TBLASTN searches ${ }^{53}$. We also conducted TBLASTN searches to identify Insig-2 genes from invertebrate genome and transcriptome assemblies, using Insig-2 protein sequences from human, western clawed frog, zebrafish, elephant shark, and Mississippi alligator as queries. Protein sequences of Insig-2 were aligned by the MUSCLE program ${ }^{54}$ to examine the presence of YECK tetrapeptide sequence. Tests of differential selection were conducted by the CODEML program in PAML $4.7^{31}$. Likelihood ratio tests were applied to compare the difference between two nested models ${ }^{31}$. The input tree of vertebrates was obtained from TimeTree (www.timetree.org).

Complete coding sequences of Insig-1 of 6 representative species of vertebrates (human, Mississippi alligator, chicken, zebrafish, western clawed frog, and elephant fish) were downloaded from GenBank. We used protein sequences of the 6 Insig-1 genes to perform TBLASTN searches against genome and transcriptome assemblies from 9 representative species of invertebrates (purple sea urchin, Pacific oyster, giant owl limpet, California two-spot octopus, sea walnut, starlet sea anemone, lamp shell, blood fluke, and sponge). In case that we found a full-length gene in a transcriptome assembly, we used this sequence as query to conduct TBLASTN searches against the corresponding genome assembly, with an aim of detecting another potential copy of this gene. Except for purple sea urchin, all remaining eight species of invertebrates examined in this study only have one copy of the Insig gene, which we named as Insig-1/Insig-2 in invertebrates. Both Insig-1 and Insig-2 genes from 6 vertebrates and Insig-1/Insig-2 genes from nine invertebrates were aligned with the MUSCLE program. Following protein sequence alignment, the nucleotide sequence alignment was generated and subsequently used to build a phylogenetic tre $\mathrm{e}^{55}$. After selecting the best-fitting model of nucleotide substitution by ModelTest ${ }^{56}$, the phylogenetic tree was reconstructed by the Bayesian approach using MrBayes v3.1.2 (ref. ${ }^{57}$ ).

Statistical analyses and reproducibility. All statistical analyses were performed using the Graphpad Prism. Data were expressed as means \pm s.d. and analyzed by unpaired two-tailed Student's $t$ test or two-way ANOVA followed by Bonferroni's post hoc analysis as indicated. Statistical significance was set at $p<0.05$. Sample sizes, statistical tests and $p$ values for each experiment are depicted in relevant figure legends

Reporting summary. Further information on research design is available in the Nature Research Reporting Summary linked to this article.

\section{Data availability}

The data that support the findings of this study are available within the paper and its Supplementary Information files, and from the corresponding author upon reasonable request.

Received: 7 October 2018; Accepted: 19 December 2019; Published online: 17 January 2020

\section{References}

1. Goldstein, J. L., Deboseboyd, R. A. \& Brown, M. S. Protein sensors for membrane sterols. Cell 124, 35-46 (2006).

2. Jiang, W. \& Song, B. L. Ubiquitin ligases in cholesterol metabolism. Diabetes Metab. J. 38, 171 (2014).

3. Sharpe et al. The UPS and downs of cholesterol homeostasis. Trends Biochem Sci. 39, 527-535 (2014).

4. Ou, Xiang. et al. Liver X receptor agonist T0901317 reduces atherosclerotic lesions in apoE-/- mice by up-regulating NPC1 expression. Sci. China C Life Sci. 51, 418-429 (2008)

5. Sever, N. et al. Insig-dependent ubiquitination and degradation of mammalian 3-hydroxy-3-methylglutaryl-CoA reductase stimulated by sterols and geranylgeraniol. J. Biol. Chem. 278, 52479-52490 (2003).

6. Song, B. L., Sever, N. \& Deboseboyd, R. A. Gp78, a membrane-anchored ubiquitin ligase, associates with Insig-1 and couples sterol-regulated ubiquitination to degradation of HMG CoA reductase. Mol. Cell 19, 829-840 (2005).

7. Song, B. L., Javitt, N. B. \& Debose-Boyd, R. A. Insig-mediated degradation of HMG CoA reductase stimulated by lanosterol, an intermediate in the synthesis of cholesterol. Cell Metab. 1, 179 (2005).

8. Miao, H., Jiang, W., Ge, L., Li, B. \& Song, B. Tetra-glutamic acid residues adjacent to Lys248 in HMG-CoA reductase are critical for the ubiquitination mediated by gp78 and UBE2G2. Acta Biochim. Biophys. Sin. 42, 303 (2010).

9. Gong, Y. et al. Sterol-regulated ubiquitination and degradation of Insig-1 creates a convergent mechanism for feedback control of cholesterol synthesis and uptake. Cell Metab. 3, 15-24 (2006).

10. Lee, J. N., Song, B., Debose-Boyd, R. A. \& Ye, J. Sterol-regulated degradation of Insig-1 mediated by the membrane-bound ubiquitin ligase gp78. J. Biol. Chem. 281, 39308-39315 (2006).

11. Ikeda, Y. et al. Regulated endoplasmic reticulum-associated degradation of a polytopic protein: p97 recruits proteasomes to insig-1 before extraction from membranes. J. Biol. Chem. 284, 34889-34900 (2009).

12. Lee, J. N., Gong, Y., Zhang, X. \& Ye, J. Proteasomal degradation of ubiquitinated insig proteins is determined by serine residues flanking ubiquitinated lysines. Proc. Natl Acad. Sci. USA 103, 4958-4963 (2006).

13. Lee, J. N., Zhang, X., Feramisco, J. D., Gong, Y. \& Ye, J. Unsaturated fatty acids inhibit proteasomal degradation of Insig-1 at a postubiquitination step. J. Biol. Chem. 283, 33772-33783 (2008).

14. Liu, T. F. et al. Ablation of gp78 in liver improves hyperlipidemia and insulin resistance by inhibiting SREBP to decrease lipid biosynthesis. Cell Metab. 16, 213 (2012).

15. Yabe, D., Brown, M. S. \& Goldstein, J. L. Insig-2, a second endoplasmic reticulum protein that binds SCAP and blocks export of sterol regulatory element-binding proteins. Proc. Natl Acad. Sci. USA 99, 12753-12758 (2002)

16. Engelking, L. J. et al. Schoenheimer effect explained-feedback regulation of cholesterol synthesis in mice mediated by Insig proteins. J. Clin. Invest. 115, 2489-2498 (2005)

17. Cadwell, K. \& Coscoy, L. Ubiquitination on nonlysine residues by a viral E3 ubiquitin ligase. Science 309, 127 (2005)

18. Wang, Y. J. et al. Cholesterol and fatty acids regulate cysteine ubiquitylation of ACAT2 through competitive oxidation. Nat. Cell Biol. 19, 808-819 (2017)

19. Miao, H., Jiang, W., Ge, L., Li, B. \& Song, B. Tetra-glutamic acid residues adjacent to Lys248 in HMG-CoA reductase are critical for the ubiquitination 
mediated by gp78 and UBE2G2. Acta Biochim. Biophys. Sin. 42, 303-310 (2010).

20. Yaffe Amp, D. \& Saxel, O. Serial passaging and differentiation of myogenic cells isolated from dystrophic mouse muscle. Nature 270, 725-727 (1977).

21. Deng, X., Mercer, S. E., Sun, C. Y. \& Friedman, E. The normal function of the cancer kinase Mirk/dyrk1B is to reduce reactive oxygen species. Genes Cancer 5, 22 (2014).

22. Ko, J. A. et al. PDZRN3 (LNX3, SEMCAP3) is required for the differentiation of C2C12 myoblasts into myotubes. J. Cell Sci. 119, 5106 (2006).

23. Poole, L. B. et al. Fluorescent and affinity-based tools to detect cysteine sulfenic acid formation in proteins. Bioconjug. Chem. 18, 2004-2017 (2007).

24. Cotgreave, I. A., Gerdes, R., Schuppe-Koistinen, I. \& Lind, C. Sglutathionylation of glyceraldehyde-3-phosphate dehydrogenase: role of thiol oxidation and catalysis by glutaredoxin. Methods Enzymol. 348, 175 (2002).

25. Schmalhausen, E. V., Nagradova, N. K., Boschi-Muller, S., Branlant, G. \& Muronetz, V. I. Mildly oxidized GAPDH: the coupling of the dehydrogenase and acyl phosphatase activities. FEBS Lett. 452, 219 (1999).

26. Schneider, C., Pratt, D. A., Porter, N. A. \& Brash, A. R. Control of oxygenation in lipoxygenase and cyclooxygenase catalysis. Chem. Biol. 14, 473-488 (2007).

27. Thomas, S. R., Witting, P. K. \& Drummond, G. R. Redox control of endothelial function and dysfunction: molecular mechanisms and therapeutic opportunities. Antioxid. Redox Signal. 10, 1713-1765 (2008).

28. Thewissen, J. G., Cooper, L. N., Clementz, M. T., Bajpai, S. \& Tiwari, B. N. Whales originated from aquatic artiodactyls in the Eocene epoch of India. Nature 450, 1190-1194 (2007).

29. Feng, P., Zheng, J., Rossiter, S. J., Wang, D. \& Zhao, H. Massive losses of taste receptor genes in toothed and Baleen Whales. Genome Biol. Evolut. 6, 1254 (2014).

30. Thewissen, J. G. M., Cooper, L. N., George, J. C., Bajpai, S. From land to water: the origin of whales, dolphins, and porpoises. Evolut. Educ. Outreach 2, 272-288 (2009).

31. Yang, Z. \& Yang, Z. H. PAML 4: phylogenetic analysis by maximum likelihood. Mol. Biol. Evolut. 24, 1586-1591 (2007).

32. Weadick, C. J. \& Chang, B. S. An improved likelihood ratio test for detecting site-specific functional divergence among clades of protein-coding genes. Mol. Biol. Evolut. 29, 1297 (2012).

33. Cupello, C., Meunier, F. J., Herbin, M., Clément, G. \& Brito, P. M. Lung anatomy and histology of the extant coelacanth shed light on the loss of airbreathing during deep-water adaptation in actinistians. R. Soc. Open Sci. 4, 161030 (2017).

34. Torrans, L. \& Steeby, J. Effects of dissolved oxygen concentration on oxygen consumption and development of channel catfish eggs and fry: implications for hatchery management. North Am. J. Aquac. 70, 286-295 (2008).

35. Silver, S., Warren, C. \& PeterDoudoroff Dissolved oxygen requirements of developing steelhead trout and chinook salmon embryos at different water velocities. Trans. Am. Fish. Soc. 92, 327-343 (1963).

36. Paramvir, D. \& Boore, J. L. Two rounds of whole genome duplication in the ancestral vertebrate. PLOS Biol. 3, e314 (2005).

37. Yabe, D., Komuro, R., Liang, G., Goldstein, J. L. \& Brown, M. S. Liver-specific mRNA for Insig-2 down-regulated by insulin: Implications for fatty acid synthesis. Proc. Natl Acad. Sci. USA 100, 3155-3160 (2003).

38. Lee, J. N. \& Ye, J. Proteolytic activation of sterol regulatory element-binding protein induced by cellular stress through depletion of Insig-1. J. Biol. Chem. 279, 45257-45265 (2004).

39. Joon No, L., Yi, G., Xiangyu, Z. \& Jin, Y. J. Proteasomal degradation of ubiquitinated Insig proteins is determined by serine residues flanking ubiquitinated lysines. Proc. Natl Acad. Sci. USA 103, 4958-4963 (2006).

40. Joon No, L., Baoliang, S., Debose-Boyd, R. A. \& Jin, Y. J. Sterol-regulated degradation of Insig-1 mediated by the membrane-bound ubiquitin ligase gp78. J. Biol. Chem. 281, 39308-39315 (2006).

41. Saltiel, A. R. New perspectives into the molecular pathogenesis and treatment of type 2 diabetes. Cell 104, 517-529 (2001).

42. Wallberg-Henriksson, H., Constable, S. H., Young, D. A. \& Holloszy, J. O. Glucose transport into rat skeletal muscle: interaction between exercise and insulin. J. Appl. Physiol. 65, 909-913 (1988).

43. Yu, T., Robotham, J. L. \& Yoon, Y. Increased production of reactive oxygen species in hyperglycemic conditions requires dynamic change of mitochondrial morphology. Proc. Natl Acad. Sci. USA 103, 2653-2658 (2006).

44. Hsia, C. C., Schmitz, A., Lambertz, M., Perry, S. F. \& Maina, J. N. Evolution of air breathing: oxygen homeostasis and the transitions from water to land and sky. Compr. Physiol. 3, 849-915 (2013).

45. MacIver, M. A., Schmitz, L., Mugan, U., Murphey, T. D. \& Mobley, C. D. Massive increase in visual range preceded the origin of terrestrial vertebrates. Proc. Natl Acad. Sci. USA 114, E2375-E2384 (2017).

46. Berner, R. A. \& Canfield, D. E. A new model for atmospheric oxygen over Phanerozoic time. Am. J. Sci. 289, 333-361 (1989).
47. Blaustein, R. The great oxidation event evolving understandings of how oxygenic life on Earth began. BioScience 66, 189-195 (2016)

48. Kiang \& American, N. Y. J. S. The color of plants on other worlds. Sci. Am. 298, 48-55 (2008)

49. Strauss, B. 10 steps of animal evolution, from fish to primates. ThoughtCo https://www.thoughtco.com/evolution-of-vertebrate-animals-4040937 (2019)

50. KJ, N. et al. Use of dimedone-based chemical probes for sulfenic acid detection methods to visualize and identify labeled proteins. Methods Enzymol. 473, 77-94 (2010).

51. Liao, Y. et al. The non-canonical NF- $\kappa$ B pathway promotes NPC2 expression and regulates intracellular cholesterol trafficking. Sci. China Life Sci. 61, 1222-1232 (2018)

52. Xiao, J. et al. Cholesterol transport through the peroxisome-ER membrane contacts tethered by PI(4,5)P2 and extended synaptotagmins. Sci. China Life Sci. 62, 1117-1135 (2019)

53. Altschul, S. F., Gish, W., Miller, W., Myers, E. W. \& Lipman, D. J. Basic local alignment search tool. J. Mol. Biol. 215, 403-410 (1990).

54. Edgar, R. C. MUSCLE: multiple sequence alignment with high accuracy and high throughput. Nucleic Acids Res. 32, 1792-1797 (2004).

55. Wei, H. \& Zhao, H. Vampire bats exhibit evolutionary reduction of bitter taste receptor genes common to other bats. Proc. Biol. Sci. 281, 20141079 (2014).

56. Darriba, D., Taboada, G. L., Doallo, R. \& Posada, D. jModelTest 2: more models, new heuristics and high-performance computing. Nat. Methods $\mathbf{9}, 772$ (2012).

57. Ronquist, F. \& Huelsenbeck, J. P. MrBayes 3: Bayesian phylogenetic inference under mixed models. Bioinformatics 19, 1572-1574 (2003).

\section{Acknowledgements}

This work was supported by grants from the NNSF of China (31690102, 31600651, 31701030, and 91753204), MOST of China (2016YFA0500100), 111 Project of Ministry of Education of China (B16036). B.-L. Song acknowledges the support from the Tencent Foundation through the XPLORER PRIZE. We thank Biyu Xiang for technical assistance.

\section{Author contributions}

B.-L. Song conceived the project. Z.-S. Zhou, M.-X. Li, J. Liu, X.-J. Shi, J. Luo, and B.-L. Song designed the experiments. Z.-S. Zhou, M.-X. Li, and J. Liu conducted the experiments. L. Chu, J. Liu and W. Qi prepared some reagents. J.-M. Xia, H. Jiao, and H. Zhao performed the evolutionary analyses. M.-X. Li, H. Zhao, J. Luo, and B.-L. Song wrote the paper with other authors' input.

\section{Competing interests}

The authors declare no competing interests.

\section{Additional information}

Supplementary information is available for this paper at https://doi.org/10.1038/s41467019-14231-w.

Correspondence and requests for materials should be addressed to B.-L.S

Peer review information Nature Communications thanks Takushi Kishida and the other anonymous, reviewer(s) for their contribution to the peer review of this work.

Reprints and permission information is available at http://www.nature.com/reprints

Publisher's note Springer Nature remains neutral with regard to jurisdictional claims in published maps and institutional affiliations.

Open Access This article is licensed under a Creative Commons Attribution 4.0 International License, which permits use, sharing, adaptation, distribution and reproduction in any medium or format, as long as you give appropriate credit to the original author(s) and the source, provide a link to the Creative Commons license, and indicate if changes were made. The images or other third party material in this article are included in the article's Creative Commons license, unless indicated otherwise in a credit line to the material. If material is not included in the article's Creative Commons license and your intended use is not permitted by statutory regulation or exceeds the permitted use, you will need to obtain permission directly from the copyright holder. To view a copy of this license, visit http://creativecommons.org/ licenses/by/4.0/

(C) The Author(s) 2020 